\title{
Analysis of Factors Affecting Energy Aware Routing in Wireless Sensor Network
}

\author{
Sohail Jabbar $\mathbb{D}^{1},{ }^{1}$ Muhammad Asif Habib, ${ }^{1}$ Abid Ali Minhas $\mathbb{D},{ }^{2}$ Mudassar Ahmad $\mathbb{D},{ }^{1}$ \\ Rehan Ashraf $\left(\mathbb{D},{ }^{1}\right.$ Shehzad Khalid ${ }^{(D)}{ }^{3}$ and Kijun Han ${ }^{(D}{ }^{4}$ \\ ${ }^{1}$ Department of Computer Science, National Textile University, Faisalabad, Pakistan \\ ${ }^{2}$ College of Computer and Information Systems, Al Yamamah University, Riyadh, Saudi Arabia \\ ${ }^{3}$ Department of Computer Engineering, Bahria University, Islamabad, Pakistan \\ ${ }^{4}$ Department of Computer Science and Engineering, Kyungpook National University, Daegu, Republic of Korea
}

Correspondence should be addressed to Kijun Han; kjhan@knu.ac.kr

Received 28 August 2017; Accepted 27 December 2017; Published 18 February 2018

Academic Editor: Houbing Song

Copyright (C) 2018 Sohail Jabbar et al. This is an open access article distributed under the Creative Commons Attribution License, which permits unrestricted use, distribution, and reproduction in any medium, provided the original work is properly cited.

Among constituents of communication architecture, routing is the most energy squeezing process. In this survey article, we are targeting an innovative aspect of analysis on routing in wireless sensor network (WSN) that has never been seen in the available literature before. This article can be a guiding light for new researchers to comprehend the WSN technology, energy aware routing, and the factors that affect the energy aware routing in WSN. This insight comprehension then makes the ways easy for them in designing such types of algorithms as well as evaluating the authenticity and extending the existing algorithms of this category, since algebraic and graphical modelling of these factors is also demonstrated. Various available techniques used by existing routing algorithms to handle these factors in making themselves energy aware are also given. Further, they are analyzed along with the suggested improvements for the researchers. At the end, we presented our previously published research work as an example and case study of discussed factors. A rich list of references is also cited for interested readers to explore the related given points.

\section{Introduction to Wireless Sensor Network}

A typical node is made up of a mote and a sensor board along with battery and a memory device. The deployment of sensor nodes is either in uniform or in random fashion that is defined by the requirements and the available situation. Their line of sight communication range is 75 to $100 \mathrm{~m}$ with a $1 / 2$ wave dipole antenna in the outdoor environment. This range is only 20 to $30 \mathrm{~m}$ with $1 / 2$ wave dipole antenna in the indoor environment [1]. They support various communication bands like $433 \mathrm{MHz}, 869-915 \mathrm{MHz}$, and $2.4 \mathrm{GHz}$ with multiple channels and provide supple solutions for various applications. The maximum data rate for signal transmission is $250 \mathrm{Kbps}$ but it depends upon the choice of configuration and the radio. The radios are half-duplex bidirectional. The Direct Sequence Spread Spectrum (DSSS) secures the data transmission. The positioning information technique like GPS-free localization [2], relative localization [3], or absolute localization [4] is used by the nodes to know about the transient nodes for communicating its data to far distant nodes or base station.

The required environmental physical quantity is sensed by the source node(s) and is disseminated through the network up-to-the data fusion centre or base station. The information so obtained can be used in Ethernet-based networks as well as worldwide by connecting the sink to the IP-based network [5]. In all of these operations, special techniques should be employed to tactfully manage the constraint resources of wireless sensor network (WSN). Among these constraint resources, energy conservation is the core issue. The major energy sources of these miniature sensor nodes are either energy storage devices like batteries or energy scavenging devices like vibration or a combination of both. Solar radiation is the most abundant energy source and yields around $1 \mathrm{~mW} / \mathrm{mm} 2(1 \mathrm{~J} /$ day $/ \mathrm{mm} 2)$ in full sunlight or $1 \mu \mathrm{W} / \mathrm{mm} 2$ under bright indoor illuminations [6]. Vibration has been proposed as an energy source that can be scavenged. So far as energy consumption is concerned, 


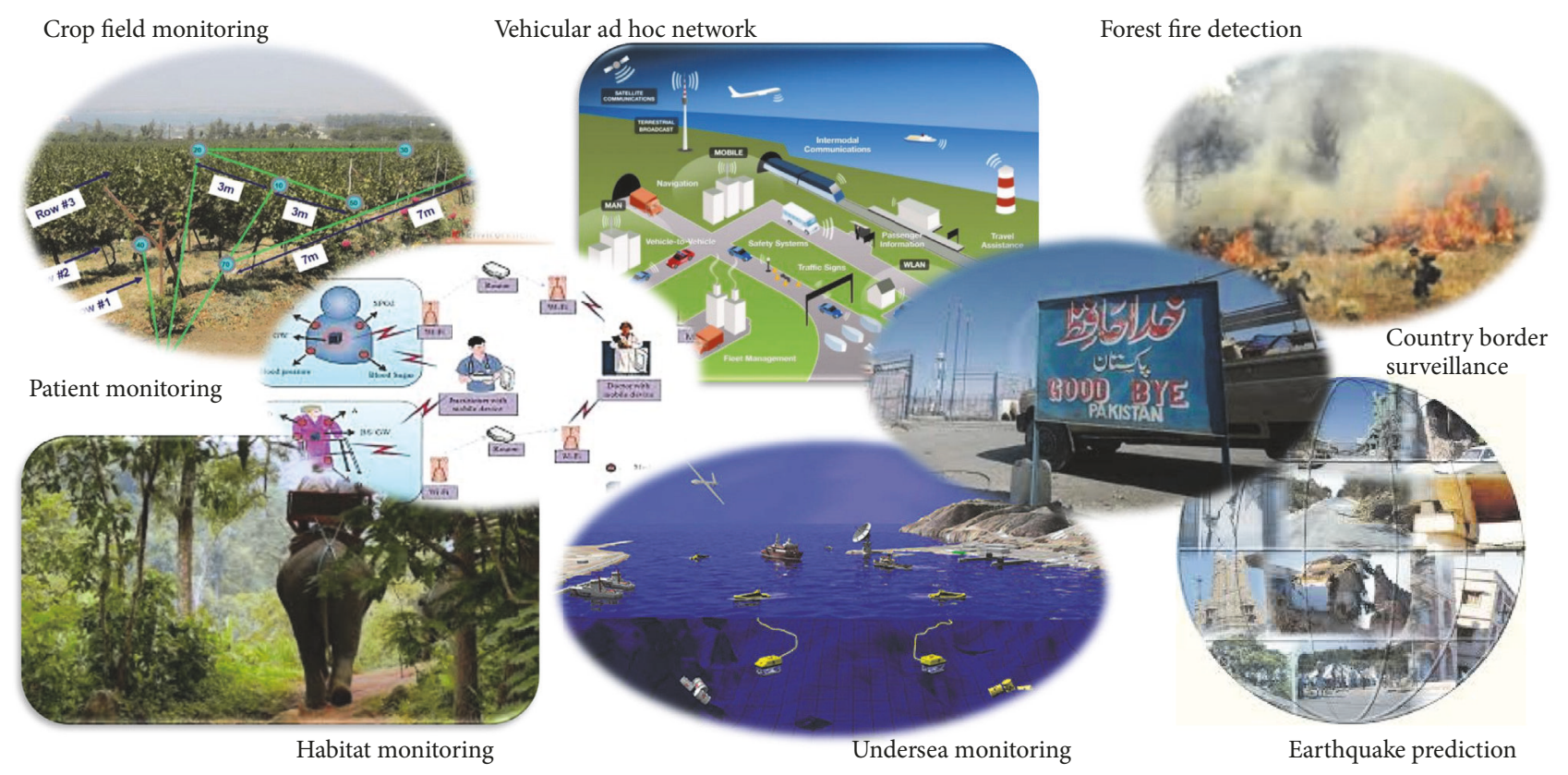

FIGURE 1: Various applications of WSN.

sensor acquisition can be achieved at $1 \mathrm{~nJ}$ per sample, and modern processors can perform computation as low as $1 \mathrm{~nJ}$ per instructions. Current transmission techniques (e.g., Bluetooth) consume about $100 \mathrm{~nJ}$ per bit for a distance of $10 \mathrm{~m}$ to $100 \mathrm{~m}$, making communication very expensive compared to acquisition and processing [6]. Stateless and light-weight protocols, dynamic power adjustment, and different power saving modes are excellent candidate solutions to minimize computation and to save transmitting, receiving, sensing, and processing power to add more life to WSN in its constraint resource environment. In wireless sensor network, nodes are uniformly or stochastically (randomly) deployed in the target region. Interconnection of nodes in a decentralized fashion without following any preexisting infrastructure is named as an ad hoc network.

1.1. Architectural Design. In wireless sensor network, the scattering of nodes in the target area is to decentralize the network traffic and balance the computational load, increasing the network coverage, reliability, and capacity. This distributed scenario of sensor nodes in the network helps in avoiding the single point of failure. This distributed computing paradigm is also enforced with various states of nodes as a sensor, router, or a gateway. This connectivity is depicted in the form of a nondirected graph with nodes as vertices and communication link as edges. The network nodes establish communication links with each other nodes in their footprint through the omnidirectional full duplex antenna. This cooperative interaction among the network nodes covers the whole network that gives birth to the selfsynchronization, self-organizing, and self-adaptive capabilities of wireless sensor network.
1.2. Real-World Applications. WSN technology that is encapsulating such a long list of qualities is now in action with lots of mind-blowing applications by indulging it in various fabrics of life. The cradle of WSN research was first nourished by Defense Advanced Research Projects Agency (DARPA) through the military applications. It continued to explore this technology by funding some prominent research projects, for example, Smart Dust [8] and Network Embedded System Technology [9]. Some other important real-world applications/projects of WSN [10] are Wave Monitoring [11], Ocean Water Monitoring [12], ZebraNet [13], Cattle Herding [14], and neuRFon [15]. This emerging technology also has its applications in Habitat Monitoring [16], Traffic Surveillance [17], Ocean Water and Bed Monitoring [18], Wildlife Monitoring [19], Cold Chain Monitoring [20], Landslide Detection [21], Vital Sign Monitoring [22], Tracking Vehicles [23], and Living and Residential Monitoring [24]. A typical WSN application at the border is shown in Figure 1. Another synergistic mating of the wireless network (for data acquisition) and a wired network (data distribution) is graphically modelled in Figure 2.

Underwater sensor network that is typically based on ultrasound is also a key application of WSN [54]. A list of various sensor network's world fame projects with their short description, related publication, and information link is given in Table 1. Other latest application based works of WSN are mentioned in $[55,56]$.

1.3. Organization of Paper. This manuscript is based on research work presented in [57]. In this paper, the under consideration topic is related to network layer for analyzing the factors that affect most of the energy aware aspect of the 
TABLE 1: Real-world WSN projects with their short description, related publications, and/or information link.

\begin{tabular}{|c|c|c|}
\hline Project & Purpose of project & $\begin{array}{l}\text { Related publication } \\
\text { and/or information link }\end{array}$ \\
\hline Sickbuilding & Wireless sensor networks in sick buildings & {$[25]$} \\
\hline Sensorscope & Sensor networks for environmental monitoring & [26-29] \\
\hline MIT River Hunduras & $\begin{array}{l}\text { Sensor networks for physical event monitoring: flood prediction in Central } \\
\text { America }\end{array}$ & {$[30,31]$} \\
\hline PermaSense & To use in remote areas with harsh environmental monitoring conditions & {$[32,33]$} \\
\hline Water for a Healthy Country & Dairy water use in Australian dairy farms & {$[34]$} \\
\hline Airy Notes & To monitor environmental condition in Shinjuku Gyoen Garden & {$[35,36]$} \\
\hline Redwood Forest & Study of California's state tree & {$[37,38]$} \\
\hline Volcanoes & Monitoring eruptions of active and hazardous volcanoes & {$[39]$} \\
\hline
\end{tabular}

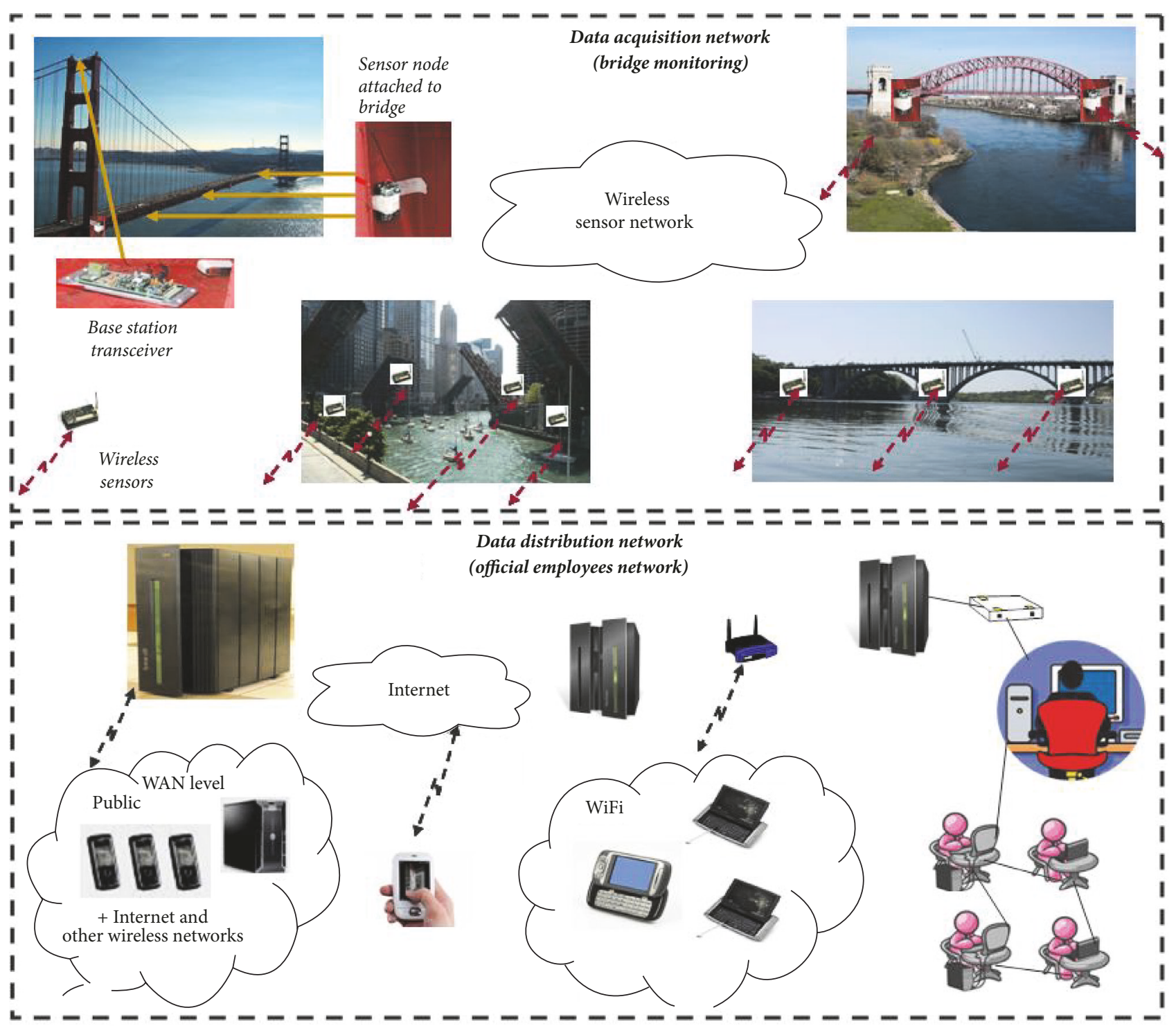

FIGURE 2: Synergistic mating of wireless network (for data acquisition) and wired network (data distribution) [7]. 
TABLE 2: List of hot research topics at different layers in WSN.

\begin{tabular}{ll}
\hline Layer & Issues \\
\hline Physical layer & $\begin{array}{l}\text { Frequency selection/carrier frequency generation, data encryption, modulation and } \\
\text { demodulation, channel coding/modelling, signal detection, antenna sensitivity and transceiver } \\
\text { design, wave propagation, spread spectrum communication, packet transmission and } \\
\text { synchronization, channel coding, multiplexing/demultiplexing }\end{array}$ \\
\hline Link/MAC layer & $\begin{array}{l}\text { Hidden node problem, congestion control, error control, medium access, radio transmission } \\
\text { power control, link quality estimation, network security, bandwidth utilization, localization and } \\
\text { positioning, time synchronization, scalability, topology control, naming and addressing }\end{array}$ \\
\hline Network layer & $\begin{array}{l}\text { Route discovery, forwarding node selection, neighbor discovery, reroute discovery, void bridging, } \\
\text { energy conservation, network security, node operational lifetime, energy efficient cluster } \\
\text { designing, throughput improvement }\end{array}$ \\
\hline Transport layer & $\begin{array}{l}\text { End-to-end retransmission based error control, event-to-sink transport reliability, sink-to-sensor } \\
\text { transport reliability, congestion control, transmission power control, effect of mobility on route } \\
\text { stability }\end{array}$ \\
\hline Application layer & $\begin{array}{l}\text { Physical topology utilization, query generation and process in different schemes of data } \\
\text { aggregation and data generation in flat or in clustered networks }\end{array}$ \\
\hline [49, 50]
\end{tabular}

routing process. Section 2 is on background study followed by Section 3 that is dedicated to routing with many subsections explaining the above target of this article. A brief discussion of the article is carried out in Section 4. Conclusion is in Section 5 and Acknowledgments section is at the end of the paper.

\section{Background Study}

This section explains the emerging research areas and energy aware routing environment in WSN. A detailed note on these aspects is given in subsequent subsections.

2.1. Emerging Research Areas in Wireless Sensor Networks. Although WSN has its deep inseparable indulgence in a variety of applications as are elucidated in previous paragraphs, yet less computing power, constraint energy, and limited bandwidth circumscribe it to attract more applications as well as hiring the existing protocols from its ancestor: ad hoc and wireless technology. The term ad hoc network is adopted by IEEE 802.11 subcommittee in the late nineties. Ad hoc network inherited some of the tribulations of wireless communication network like unreliable time invarying asymmetric channel, improperly defined coverage boundary, lossy link, and so on. In addition to this, location awareness, multihop environment, dynamically changing topology, node, and channel vulnerability issues in ad hoc network environment have their messy contribution in this knotted portfolio, to lengthen this tribulations list. So, by the disparities mentioned above, algorithms designed for wireless networks and ad hoc networks (LAR [58]) are not suitable for WSN and need customization and improvement. So the simple wireless protocols cannot be adopted by ad hoc network without customization due to the above grounds. Similarly, wireless sensor network also comes up with some differences from its close ancestor, ANET (ad hoc network) like densely and randomly deployed nodes, unattended operation for a long period, and other constraint resources. Also in WSN, in addition to most of the aforementioned issues, network partitioning, localization, calibration, data fusion, aggregation and dissemination, coverage issues, self-organizing and selfadministration, scalability, load balancing, node clustering, topology management, end-to-end delay constraint routing, security and privacy, heterogeneity, and other energy, memory, power, and bandwidth constraints are the active challenges. In the closer view, node scheduling, hole problem, avoiding and coping with void node areas, node failure, and QoS relating factors are under the great concentration of researchers [59]. From sensing to receiving at base station (BS) through communication and processing, the entire network ingredients, their interaction, and functionalities are divided into five different layers model that is the hybrid of OSI seven layers and four layers of TCP/IP layers models [60]. Each layer has its long list of functionalities and related issues inviting the researchers to engage themselves to work on for their better performance leading to the best. Table 2 demonstrates the five working layers of WSN, their related issues, and the references of key survey articles thereon. Apart from this five layered architecture, the middle layer or cross-layer issues also have their distinguished importance and play a vital role in an efficient network performance. Each aforementioned layer has its key inseparable role in the network functionalities ranging from physical environmental sensing to generation of suitable binary bit stream, from end-to-end link reliability to error detection and correction, from node addressing to packet routing, from medium access to safe and secure transmission, from signal generation to modulation and multiplexing and ultimately dissemination of transmitted data to the destination through transient node.

\subsection{Energy Aware Routing Environment in Wireless Sensor} Networks. Each of these network's logical ingredients is interrelated and indispensable for a safe, secure, reliable, effective, and efficient communication between the interacting nodes of near or far distant networks. All of these logical ingredients are measured and finalized at the node and 

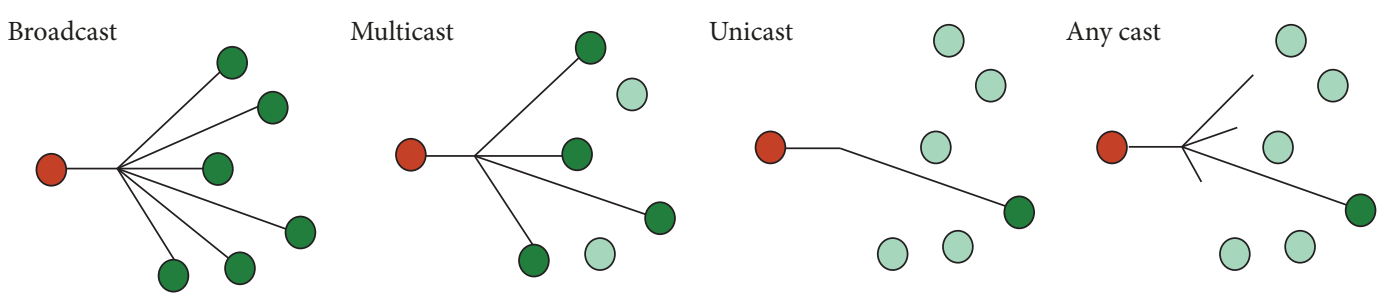

FIGURE 3: Routing schemes.

utilize its resources. Furthermore, wireless sensor network is a synergistic mating of wireless communication, sensor, and network technology. Apart from other inherited issue from the wireless and ad hoc network as mentioned in the previous section, induction of ideas in WSN is confined by its stringent constraint factors of computational power, memory, and bandwidth. Along with these, node energy is another limitation in the domain of WSN. The cause of this pervading issue is in-general battery operated and unattended deployment of WS nodes. Also, replenishment of battery is almost impossible due to inaccessible and far distant deployment of nodes in most of the applications. Since the node energy is the most participating factor in the completion of any task relating to the above issue, any of the presenting solutions in any aspect of WSN domain considers this very factor in its idea. Categorizing all the functions performed in/by WS node comes up with sensing, computation, reception, and transmission. Energy consumption in the transmission of a bit to $10 \mathrm{~m}$ to $100 \mathrm{~m}$ is equal to energy consumption in acquisition and computation of 100 bits. Hence, it is confirmed that transmission is the hungriest function for energy that is dealt by MAC layer and network layer. At MAC layer, transmission is only involved in safe access of medium while, at the network layer, transmission is the roundabout of routing process that is the key function of the network layer. Increased communication distance and bigger packet size add more energy cost in transmission function. Transmission, computation, and reception are largely involved in maintaining network table, route discovery, reroute discovery, data collection, broadcasting, forwarding node selection, and so on. Hence, acknowledging the importance of all other layer's functionalities, the role of the network layer is direly highlighted in data communication process among other four layers. Given all the above discussion, efforts are put, and ideas are floated to conserve the precious constraint resource of energy at this layer through minimum broadcasting, smart forwarding node selection mechanism, stateless path finding, route maintaining, reroute discovery algorithms, and so on. Decreasing the transmitted packet size using data fusion and data aggregation techniques is another effort to conserve the energy at the network layer. To have a closer look, the key factors at network layer that affect routing process and must be considered during the design of routing algorithm are broadcasting, beacon message exchange, probe messaging, route discovery, forwarding node selection, reroute discovery, void bridging strategies, frequent updating of neighbor table, and routing table. In case of clustered network, key factors are cluster designing, cluster head selection, cluster head rotation, cluster redesigning, forwarding $\mathrm{CH}$ selection, node level calculation/processing, and so on. In case of a mobile ad hoc network, conversing on energy in the above constituents of routing process becomes more challenging as well as adding other dynamic factors like considering the dynamic topology. The routing protocols considering the above factors ultimately play a vital role in prolonging the network lifetime. In the subsequent section, we will present the analysis of these factors mentioned above in detail.

\section{Analysis of Factors Affecting the Energy Aware Routing Function of Network Layer}

In this section, the key factors among the points above in the previous section are briefly explained along with graphical representation for the better comprehension.

3.1. Broadcasting. Broadcasting is the process of communicating the message to the nodes in footprint in one transmission. It means that the transmission of a node, $N_{i}$, is received by all the nodes, $N_{j}$, in the radio range of $i$ th node $\left(r_{i}\right)$. It can be represented as $\left|D\left(N_{i}-N_{j}\right)\right| \leq r_{i} \forall_{j}$ Where $j=$ $1,2, \ldots, 6$. The neighborhood range can be increased or decreased with dynamic power adjustment, that is, increasing or decreasing the communication power. It can be translated as the number of neighborhood nodes is the increasing function of transmission power. Although in wireless networks this categorization of routing schemes as broadcast, unicast, multicast, or any cast is set at the recipient level with respect to its targeted listeners, yet all the neighbors receive the transmission under the promiscuous receive mode, so always broadcast but decoding of the packet is only done by the node(s) whose address(es) is/are encapsulated in the packet at the recipient field. This makes the transmission broadcast (for all recipients), unicast (only one among recipients), multicast (as specific group among recipients), and any cast (any one from mentioned group among the packet recipients). Figure 3 depicts aforementioned routing schemes [61].

In most of the jobs during the routing process, the need arises to collect the neighbor information to know the nodes' status (alive or dead), to communicate nodes' parameters (energy, memory, and nodes' id), and so on through probe messages (an empty message to simply see whether the destination actually exists). In computer networks, Ping is a common utility that is used for sending such a probe or 


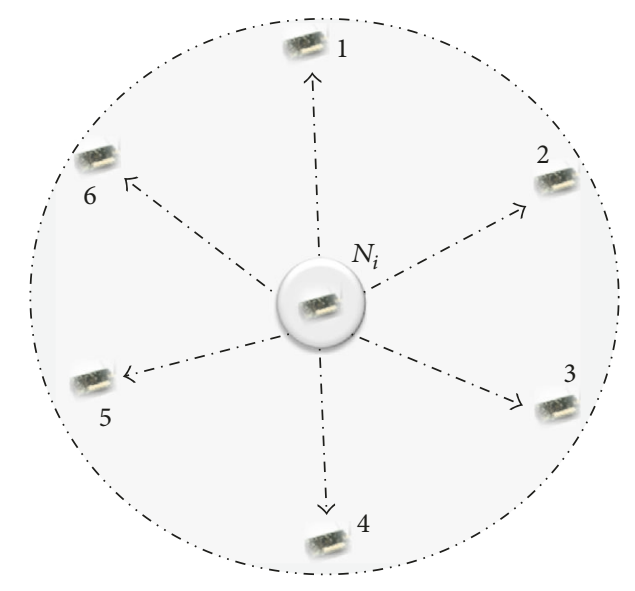

FIGURE 4: Broadcasting \beacon message exchange.

beacon message. Piggybacking is a very common technique for updating the neighbor node about node status or query some neighbor node by exploiting the control packet. This can also be broadcast, unicast, multicast, or any cast. Due to these reasons of "always broadcast nature" of messaging in wireless communication, broadcasting/beacon messages are being discouraged unless direly needed. These not only create congestion and increase end-to-end delay, to affect the routing and hence network efficiency, but also consume too much energy in receiving, demodulating, decapsulating, processing, encapsulating, modulating, and transmission processes.

Although broadcasting is the severe rivalry of energy aware routing, it is the vital part of most of the routing features like cluster formation, cluster head election, cluster head rotation, path establishment, forwarding node selection, setting up a neighbor table and routing table, path maintenance, and so on. Due to its core importance in these functionalities, ideas are floated, and solutions are proposed to minimize message broadcasting [62] and beacon message exchange [63]. A routing algorithm with minimum broadcast in the stringent constraint energy factor environment is appreciated in the research community. The straightforward approach of the broadcast to establish the route is blind flooding [64], in which each node is obligated to rebroadcast the packet whenever it receives a packet for the first time. Blind flooding generates many redundant transmissions. These redundant transmissions may cause a serious problem, referred to as the broadcast storm problem [65], in which redundant packets cause communication congestion and contention. A common approach for the remedy of broadcast storm problem is packet sequencing. When a node has packets to broadcast in the network, the broadcast protocol should route these packets to all nodes in the network with little overhead, latency, and consumed energy.

Graphical representation of this process is given in Figure 4 .

3.2. Forwarding Node Selection. The node that makes way for a node to communicate its packet to other nodes in a network or the base station is called forwarding node and the process of finding such node is referred to as forwarding node selection. According to Wu and Lou [66], in a broadcast process, the source and a subset of nodes form a flooded tree such that any other node in the network is adjacent to a node in the tree. Nodes on the tree are called forward nodes and form a connected dominating set (CDS). Forwarding node is/must be in the footprint of the current node. Forwarding node selection is the key step in route discovery, reroute discovery, and void bridging. For these to complete, the efficiency of any routing protocol depends mostly on the selection of a node that may lead its data to the destination. In case of clustered network architecture, forwarding node for a cluster member (CM) node can be cluster head $(\mathrm{CH})$ or other $\mathrm{CM}$ nodes and then the $\mathrm{CH}$ depending upon the communication scenario. The selection parameter can be energy, distance to destination (BS), nodal density (node degree), feasible position, time to reach to $\mathrm{BS}$, hop count to $\mathrm{BS}$, or the combination of these. So, a sensible decision for selecting a most suitable node for forwarding the message/data to the targeted destination node matters a lot in the success of any routing application. Its importance gets more priority when there is a concern of real-time application. Given all its importance, an effective and accurate forwarding node selection is given the proper resources and special concentration that ultimately results in more energy consumption and hence making the routing protocol less energy efficient. The selection parameters for forwarding node vary with the intended application, but in all cases, energy conservation is/should be considered, since energy is the stringent constraint factor in wireless sensor network. Moreover, a better tradeoff is also required among the competing selection parameters [67]. For example, in real-time application energy, time and distance to destination (BS) are the competing factors [68]. Wu and Lou have contributed their rich article in the literature [66]. This article targets the combined effect of broadcasting and forwarding node in designing the protocols for networks with scarce resources. The ad hoc and wireless sensor network can be the examples of such networks. The taxonomy of broadcast 


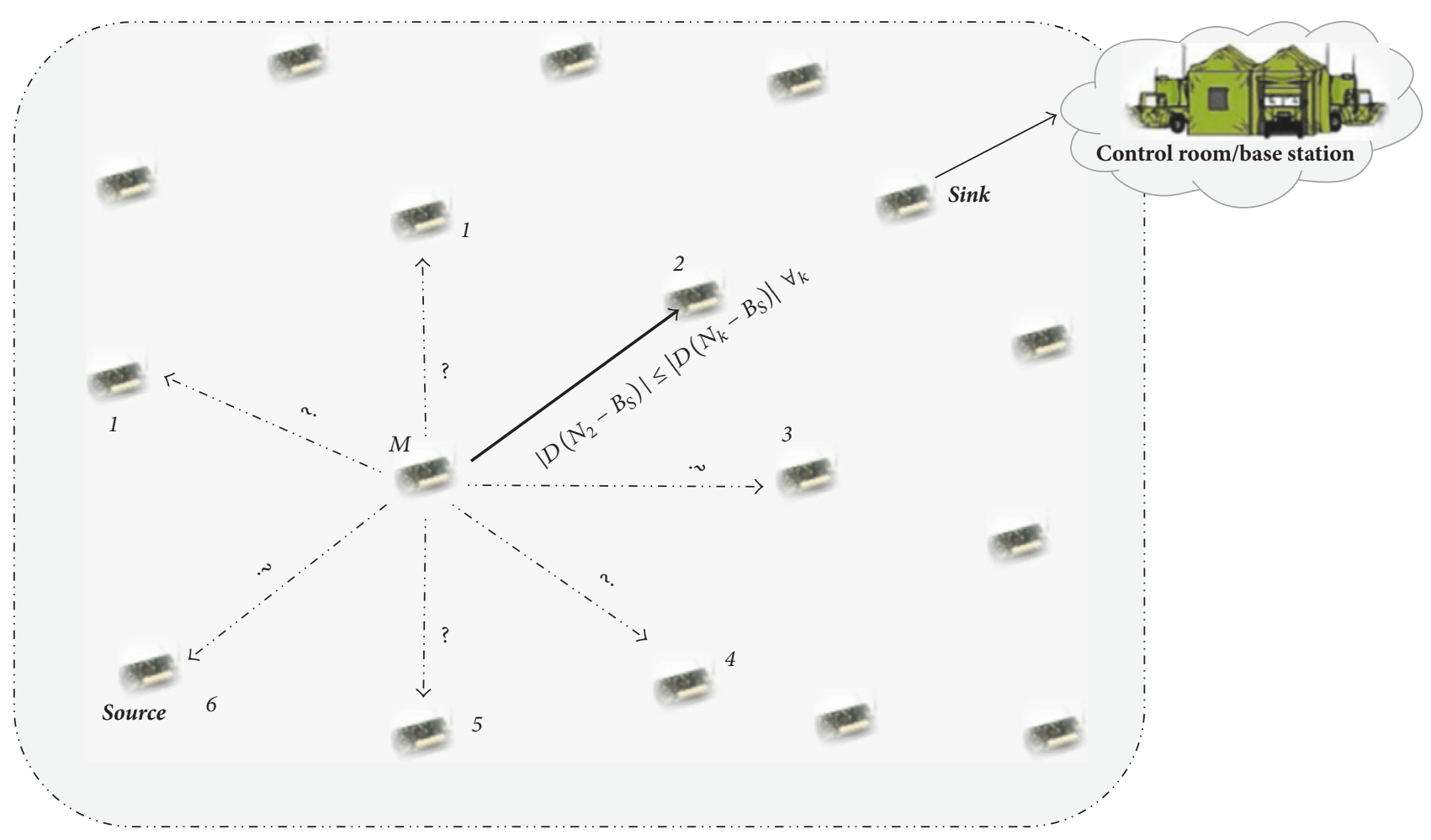

FIGURE 5: Forwarding node selection process/route discovery process.

routing protocols and nature of algorithms is also given in the article.

There is a node " $M$ " in the deployed network. All the nodes $\left(N_{j}\right)$ which are in the foot print $\left(r_{m}\right)$ of node (" $M$ ") are assumed to constitute the forwarding candidate neighbor set (FS). If the distance to base station $D\left(B_{S}\right)$ is the selection criteria for forwarding node then the node $\left(N_{j}\right)$ which has the least distance to base station will be the most favorite for being selected as forwarding a node. It can also be represented as follows:

$$
\begin{aligned}
& \left|D\left(M-N_{j}\right)\right| \leq r_{m} \quad \forall_{j} \text { Where } j=1,2, \ldots, 6 \\
& \left|D\left(N_{j}-B_{S}\right)\right| \leq\left|D\left(N_{k}-B_{S}\right)\right| \\
& \qquad \forall_{k} \text { Where } k=1,2, \ldots, 6, j \neq k .
\end{aligned}
$$

Figure 5 demonstrates this process graphically.

3.3. Route Discovery. The ultimate purpose of any routing protocol in wireless sensor networks is to bring the sensed/generated data from the source node to the sink node. In some cases, usually in the small flat network, this data is directly sent to the BS so no case of route discovery at all. If we consider this scenario in the cluster-based architecture, the sensed data is communicated either directly [69] or through multihop to the $\mathrm{CH}$ [70] which is further sent either directly to the BS [71] or through multihop [69]. In the other case that is most prevailing in large-scale flat and cluster-based WSNs due to the limitation of communication range of the node, the data is transmitted from a source to sink through transient nodes (forwarding node). The better selections of these transient nodes accumulate to a better route to the base station. Less energy consumption, minimum end-toend delay, minimum path length (hop count or distance), less number of transient nodes, better value for performance metric calculation, or combination of these are the common characteristics of a favorable and effective route [72-74]. Precedence level of these characteristics varies with targeted application. Some can be compromised at the cost of others. As an example, in a real-time application, the end-to-end delay has the highest precedence, and it must be achieved even with the cost of energy. In the competing factors, tradeoff should be set at some breakeven point. For example, among the three paths, $P_{1}, P_{2}$, and $P_{3}$, from source node $S_{o}$ to the destination node $D_{o}, P_{1}$ has less number of transient nodes $\left(T_{n}\right)$ but it is lengthy $\left(P_{L}\right) . P_{2}$ has some transient nodes with lesser length from source $S_{o}$ to the destination $D_{o}$ as compared to $P_{1} . P_{3}$ has some transient nodes and path length that is an average of both paths $P_{1}$ and path $P_{2}$; that is, $T_{n}\left(P_{1}\right)<T_{n}\left(P_{3}\right)<T_{n}\left(P_{2}\right)$ and $P_{L}\left(P_{2}\right)<P_{L}\left(P_{3}\right)<P_{L}\left(P_{1}\right)$.

Hence, Path $P_{3}$ has the characteristics that are at the tradeoff point. So, it should be selected to route the data. The route discovery process is modelled in the same way as that of forwarding node selection. Figure 5 demonstrates this process graphically.

3.4. Reroute Discovery. An extension of route discovery process is reroute discovery. Death or malfunctioning of nodes may result in breaking of the established path and may lead to network partitioning. So the need arises to 


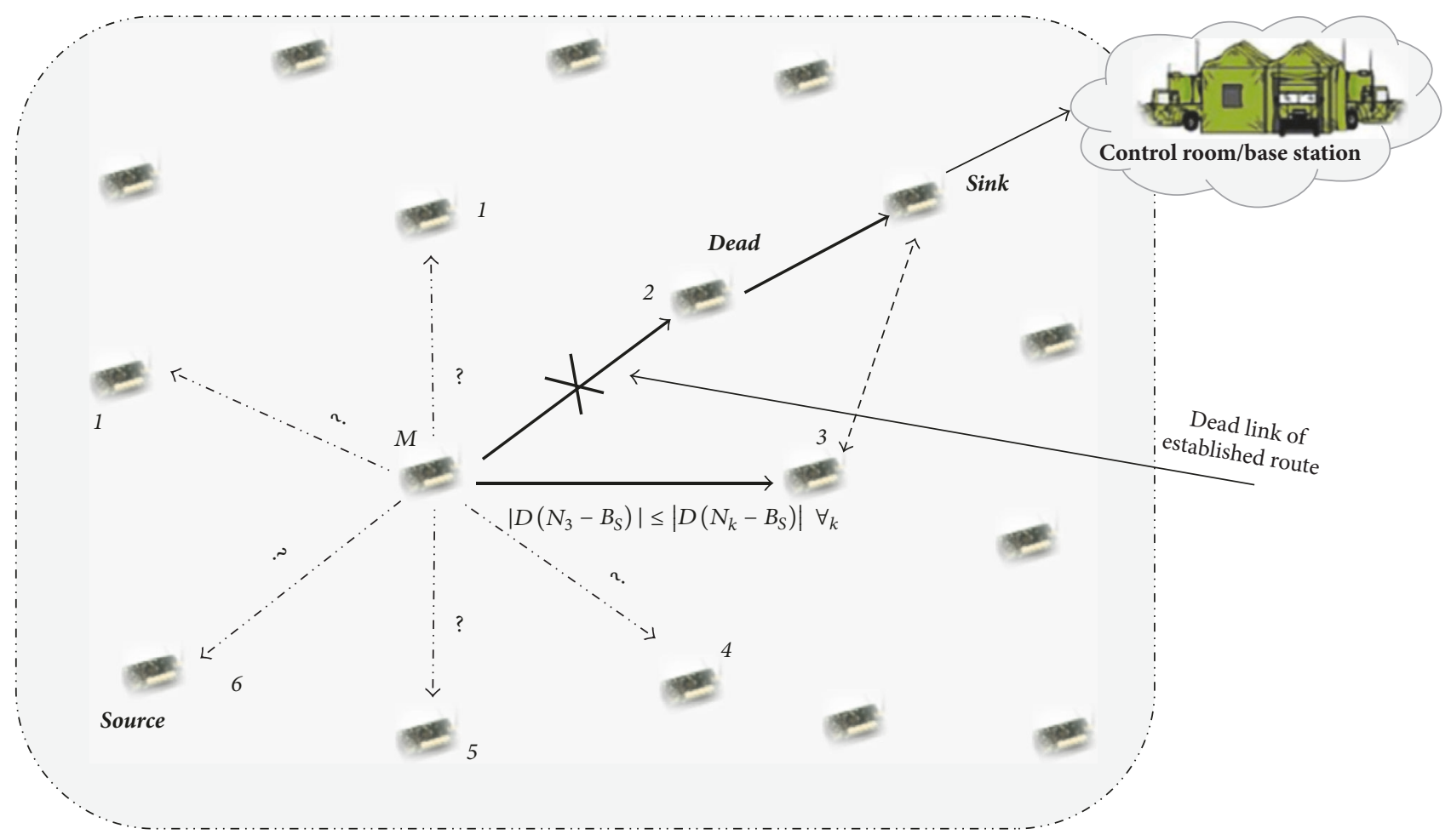

FIGURE 6: Reroute discovery process.

discover the new path. This path setting up phase that is initiated due to the breaking of the established path is called reroute discovery. This process is almost same as that of route discovery that has been discussed in very previous subsection just with a difference of initiating cause. Moreover, forwarding node selection is also an inseparable part of route discovery process. Death of node can be identified by the following methods:

(1) If, for a specific period " $t$ ", no reply of query packet is received from the relay node/forwarding node, then it is assumed that there is no more existence of this node.

(2) If for a specific period " $t$ " transmission acknowledgment from the destined node is not received, then it is considered dead.

(3) In an established path, if the expected message is not received from the specific node for a defined time and defined repeated requests, then the node is considered being either trapped or dead.

So in such situations, reroute discovery process is initiated to reestablish the disconnected path. This rout failure remedy is more frequently needed in mobile ad hoc network (MANET) [75] as compared to static ad hoc network [74].

There is a node " $M$ " in the deployed network. All the nodes $\left(N_{j}\right)$ which are in the foot print $\left(r_{m}\right)$ of node (" $M$ ") are assumed to constitute the forwarding candidate neighbor set (FS). If the distance to base station $D\left(B_{S}\right)$ is the selection criteria for forwarding node, then the node $\left(N_{j}\right)$ which has the least distance to base station will be the most favorite for being selected as forwarding node except $N_{2}$ that is already dead and due to which the reroute discovery process is initiated. It can also be represented as follows:

$$
\begin{aligned}
& \left|D\left(M-N_{j}\right)\right| \leq r_{m} \quad \forall_{j} \text { Where } j=1,2, \ldots, 6 \\
& \left|D\left(N_{j}-B_{S}\right)\right| \leq\left|D\left(N_{k}-B_{S}\right)\right| \\
& \quad \forall_{k} \text { but } j \neq 2 \text { Where } k=1,3,4,5,6, j \neq k .
\end{aligned}
$$

Figure 6 demonstrates this process graphically.

A special case of reroute discovery arises:

(i) When the established path is disconnected due to the death of forwarding node.

(ii) When, among the neighboring nodes, distance to the destination is more than the current node's distance to destination.

Then it creates a gap called void and may result in network partitioning (a scenario where a network is divided into two or more than two parts, those ever were one part). Finding a route to fill this gap is called a void bridging. Moreover, voids are also formed due to the random deployment of the nodes which raises the aforementioned second issue. In Figure 7 the forwarding node " 2 " in the path from node " $M$ " to sink has gone dead that was the only node in the forwarding candidate neighbor set (FS) of node " $M$." So, node " $M$ " is stuck since it has no neighbor that is so close to be selected as the forwarding node because its FS is empty. Hence, no 


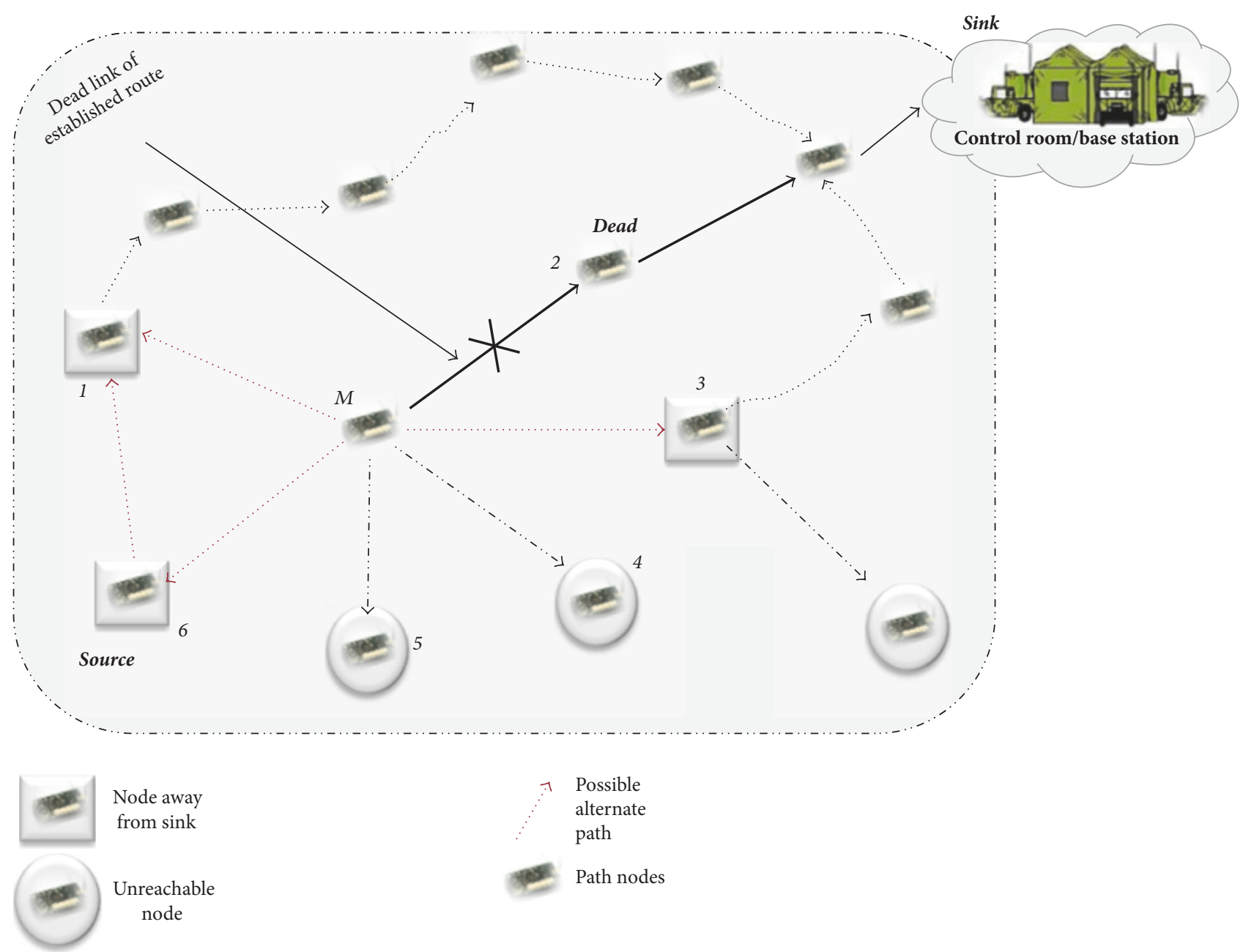

FIGURE 7: Void bridging process.

data can be positively progressed to the sink at this node. To bypass this situation (void), packet is forwarded in a negative progression using the local minimum phenomenon [76]. Let node " 3 " in Figure 7 be selected; even it is away from sink and not from the FS to lead the packet towards sink. So, the appropriate void handling approach is important in both flat and clustered network to save the node's resources (energy, computation) as well as the communication or packet to be lost. Such behavior is direly discouraged in the time-critical applications since network mission can be severely affected by the loss of some captured information [77]. This can be bridged by right-hand rule [78] or by backtracking the path and find the route to the destination by linking with some other established path [79] or by establishing a completely new path. The same process is followed to established new route as is for route discovery or reroute discovery. In such scenarios, multipath to the destination is a feasible technique. However, it has its disadvantages due to multipath finding and managing. Although the route found in such a way of backtracking is lengthy and can be infeasible, yet it saves the data to be lost continually and the network from partitioning.
3.5. Neighbor Table. The nodes those come within the footprint of node " $M$ " are considered its neighbors and the format in which and where information of these neighboring nodes is kept in the node " $M$ " is called neighbor table. Its size is increasing function of number of neighboring nodes which is further directly proportional to the transmission power [80]. In the dense network (where the number of nodes per unit area is more than usual or in other words where the distance between nodes is far less than usual), its size is bigger as compared to the sparse network [81] (where the number of nodes per unit area is less than usual or in other words where the distance between nodes is far more than usual). In memory constraint environment, such as that of WSN, keeping the information of all the neighbor nodes is a bit challenging task especially when the deployment is dense. So, different strategies are adopted as given as follows:

(1) A threshold value of the parameter (energy, memory, node density, workload, queue size, etc.) is defined, and the neighboring nodes whose values meet that defined threshold criteria are kept in the neighbor table [72]. 


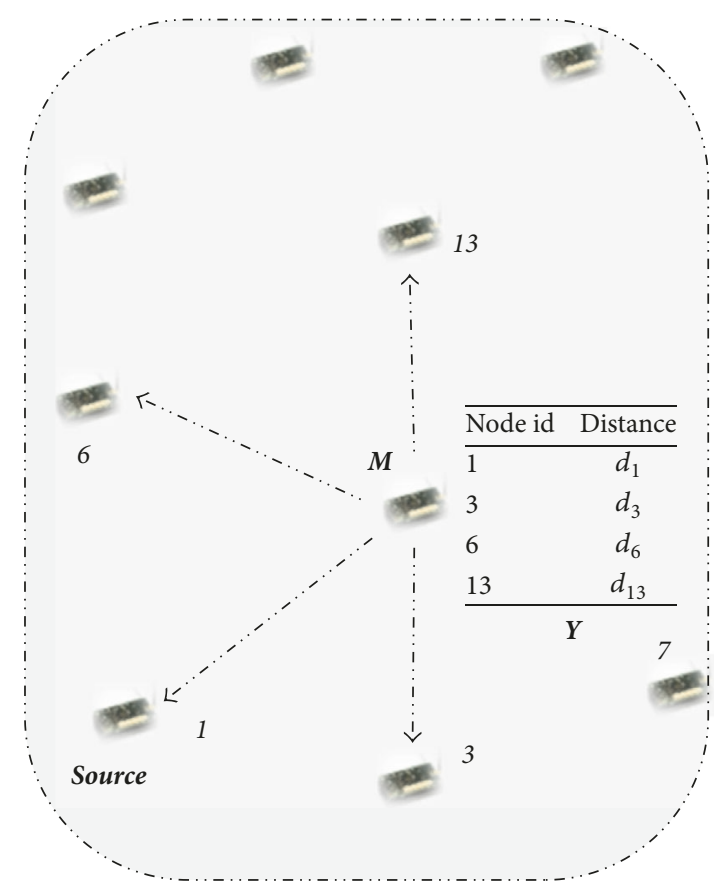

FIgURE 8: Neighbor table designing process.

(2) Among all the nodes in the neighbor that have the redundant data, only one node's information is added in the neighbor table [82].

Many routing algorithms need a neighbor table of the current node [72] and the neighboring nodes to start functionality or to complete their task. Adapting the same strategies help in energy conservation during communication due to less size. This requires a neighbor table (i) to be updated, (ii) to be communicated, and (iii) to be stored, in one case or the other. Each of these three requirements has their separate challenges to be satisfied and have their optimistic or pessimistic contribution in different algorithms designed for various applications.

Moreover these three functions directly or indirectly may result in (i) network congestion, (ii) increased packet drop ratio, (iii) increased end-to-end delay, (iv) increased packet size, (v) increased computation, and (vi) decreased throughput and the most important are (vii) more energy consumption and (viii) decreased network lifetime. So, the well-planned algorithm in this context is indispensable for successful energy aware routing protocol for wireless sensor network.

There is a node " $M$ " in the deployed network and a neighbor table " $Y$ " at node " $M$ " which contains all the nodes whose distance is less than the radio range of node " $M$." So in Figure 8 neighbor table contains the information of node id $\left(N_{j_{\text {id }}}\right)$ and node's distance $\left(N_{j_{\text {Dist }}}\right)$ from node " $M$." This can be represented as follows:

$$
\exists_{M} \exists_{Y}
$$

$M=$ Node, $Y=$ Neighbors of $M$ in neighbor table

$$
\begin{aligned}
& \operatorname{At}(M: Y) \Lambda N_{j} \in Y,\left|D\left(M-N_{j}\right)\right| \leq r \\
& \quad \forall_{j} \text { Where } j=1,3,6,13, N_{j} \ni\left(N_{j_{\text {id }}}, N_{j_{\text {Dist }}}\right) .
\end{aligned}
$$

3.6. Routing Table. The constituent nodes that assist a packet to direct to the destination node(s) make the route, and those constituent nodes of those routing paths are called the route nodes. The table that keeps track of those route/path nodes in a specific format from current nodes to the destination nodes (sink node/BS) is called the routing table. The table may contain various attributes of route nodes from node id, node energy level, congestion rate, packet drop ratio, memory and distance from sink depending on the routing algorithm, target application, or decision requirements [83]. In some of the cases, routing table needs to be communicated, but its regular update is necessary in almost all cases. Memory constraint is also a factor that is to consider in managing this aspect of routing. These all have their strong impact not only on the efficient routing positively but also on its energy consumption aspect negatively. This severity becomes more intense in case of a large-scale network. Hence big routing table squeezes more network energy along with pessimistically affecting other network parameters. Since the cluster-based network is more organized than flat based sensor network, establishing and maintaining the updated routing table in the clustered network are comparatively less costly than in flat networks. Due to these very reasons, exchange of routing table is not appreciated to be included in routing algorithm unless direly needed in special case of routing which is affiliated to the requirements of routing 


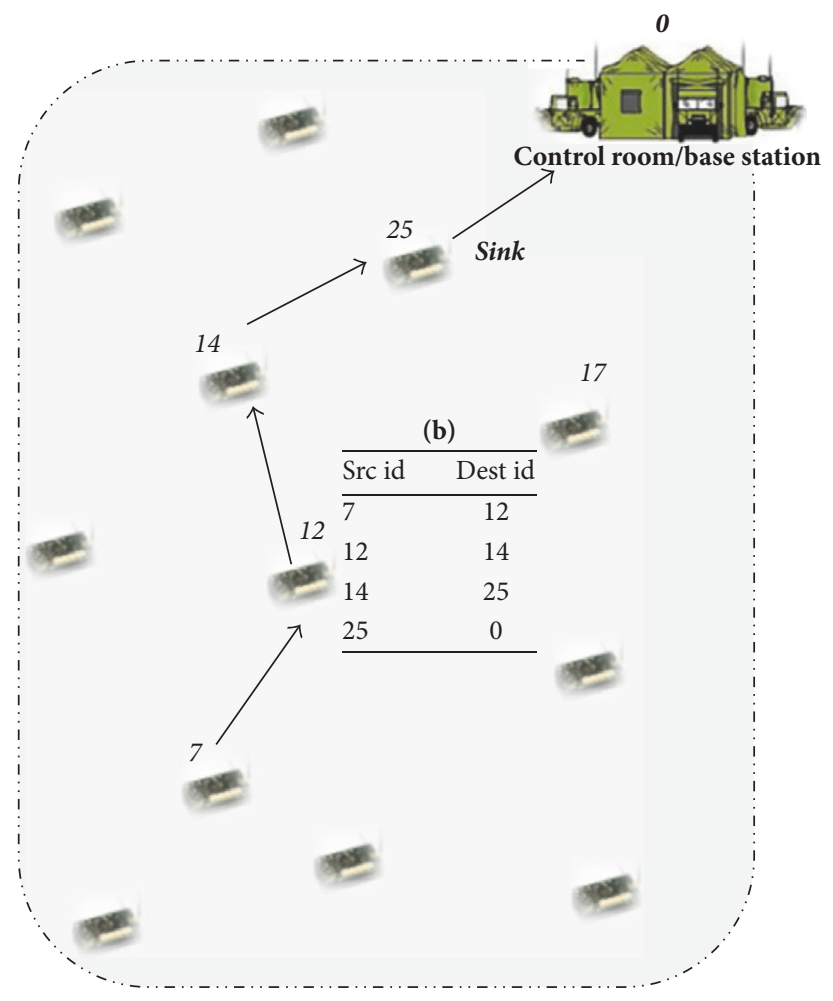

Figure 9: Routing table designing process.

algorithm or underlying application. Introducing the routing table is more common in the static wireless sensor network (SWSN) as compared to mobile wireless sensor network (MWSN). Its management is a big challenging task and in some cases almost impossible as in the scenario with high mobility of nodes. Routing table designing strategies, its requirements, and its challenges are almost same as that of the neighbor table mentioned previously in the same section with some tailoring effects.

Let, in a network of " $n$ " nodes, $X$ and $Y$ be the sources and destination nodes and $i$ and $j$ be intermediate nodes with node ids 7, 25, 12, and 14, respectively. Routing table " $R$ " at node " $X$ " has the information of complete path " $P$ " that is comprised of links $(\rightarrow)$ from source node to destination node passing through the intermediate nodes in such a ways that each next node, say " $i$," is in the radio range " $r$ " of the previous node, say " $X$." This is represented as $\mid D(X-$ $i) \mid \leq r$. A simple representation of this scenario is given as follows:

$\exists_{X}, \exists_{X}, \exists_{R}$

$X=$ Source Node, $Y=$ Destination Node, $R=$ Routing Table.

At $(X: R)$,

$$
\exists_{i} \exists_{j} \quad i, j=\text { Intermediate Nodes, }
$$

where $|D(X-B S)|<|D(i-B S)|<|D(j-B S)|<|D(Y-B S)|$.

$$
\begin{gathered}
\text { Moreover, } L_{1}=X \rightarrow i, L_{2}=i \rightarrow j, L_{3}=j \rightarrow Y \\
R=L_{1}+L_{2}+L_{3}, \\
|D(X-i)| \leq r_{X}, \\
|D(i-j)| \leq r_{i}, \\
|D(j-Y)| \leq r_{j} .
\end{gathered}
$$

The same is depicted in Figure 9.

3.7. Network Clustering. Clustering is the function of grouping nodes in a centralized way. The nodes should be autonomous where one node is treated as head and other nodes are treated as members. The group is named as a cluster. The nominated head is called cluster head $(\mathrm{CH})$ and other nodes in the role of members are called cluster members (CM). The node that is not yet a part of any cluster will be called undecided node. The $\mathrm{CH}$ gets the data that was being sensed by sensors and sent via CMs. It then aggregates and forwards this data to other CHs. This can be sent to base station directly (direct-hop) or through some temporary node or gateway node (multihop). Thus the nodes are being assigned various roles in a clustered network architecture. In Figure 10 all possible states of a node during its lifetime are being shown depending upon the clustering algorithm. In the figure the underlined states are considered the part of every clustering algorithm. 


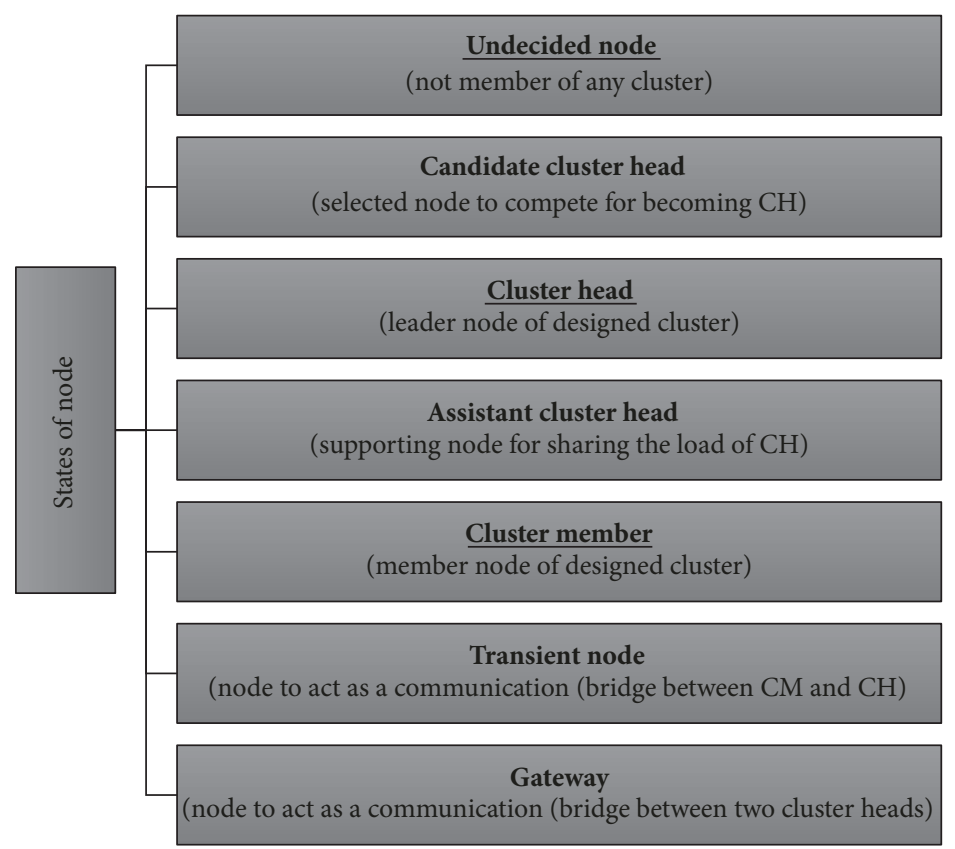

FIGURE 10: States of a node in clustered network architecture.

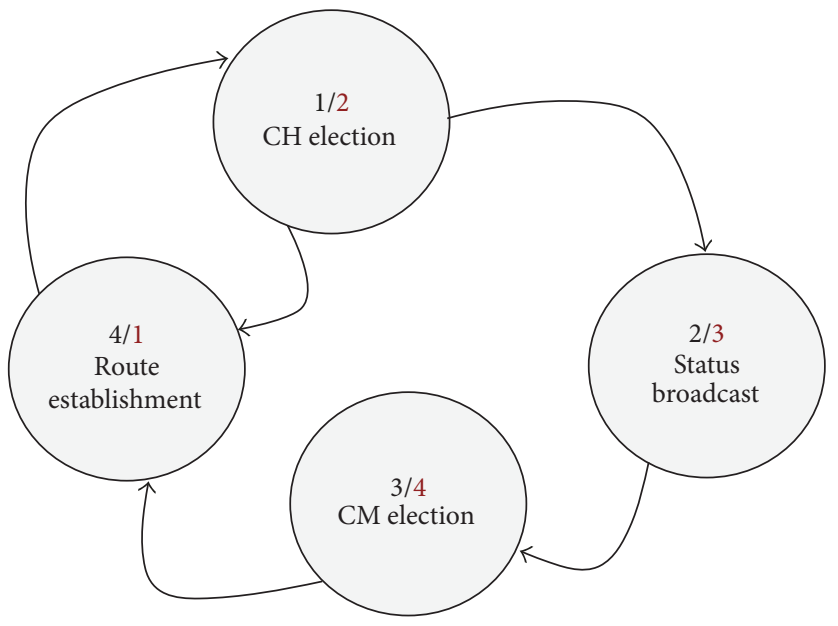

FIGURE 11: Steps in constitution of clustered network.

The process of routing is divided into two categories. One is intracluster and other is intercluster data transmission. The intracluster means that the data transmission will be within cluster that depicts that it will be from cluster member node to cluster head. The intercluster means that the data transmission will be between clusters that depicts that it will be from $\mathrm{CH}$ from one cluster to the $\mathrm{CH}$ from other cluster. This data transmission method enables us to save significant energy. The nodes in the network who have the same configuration parameters, that is, energy, power, computation, and memory, are called homogenous nodes. The nodes who have different configuration parameters are called heterogeneous nodes. When a clustered network is designed the first step is the selection of $\mathrm{CH}$, that is, designating a node as a cluster head in the deployed network. The last step is the received data from deployed nodes in the process of establishing the route for communication [69]. Figure 11 elaborates the phases involved in the composition of the clustered network.

The clustered network in the architectural setup has self-support in the preservation of energy. In this type of network an appropriately positioned cluster head that works as gateway and high energy shows a key role in the solution of said purpose [84]. The architecture of clustered network due to its ease of route discovery, data aggregation, fault tolerance, and end-to-end delay is considered as the most energy efficient [85]. The clustered network architecture is proved to be energy aware routing but there is a need to make it better in energy consumption especially in the 


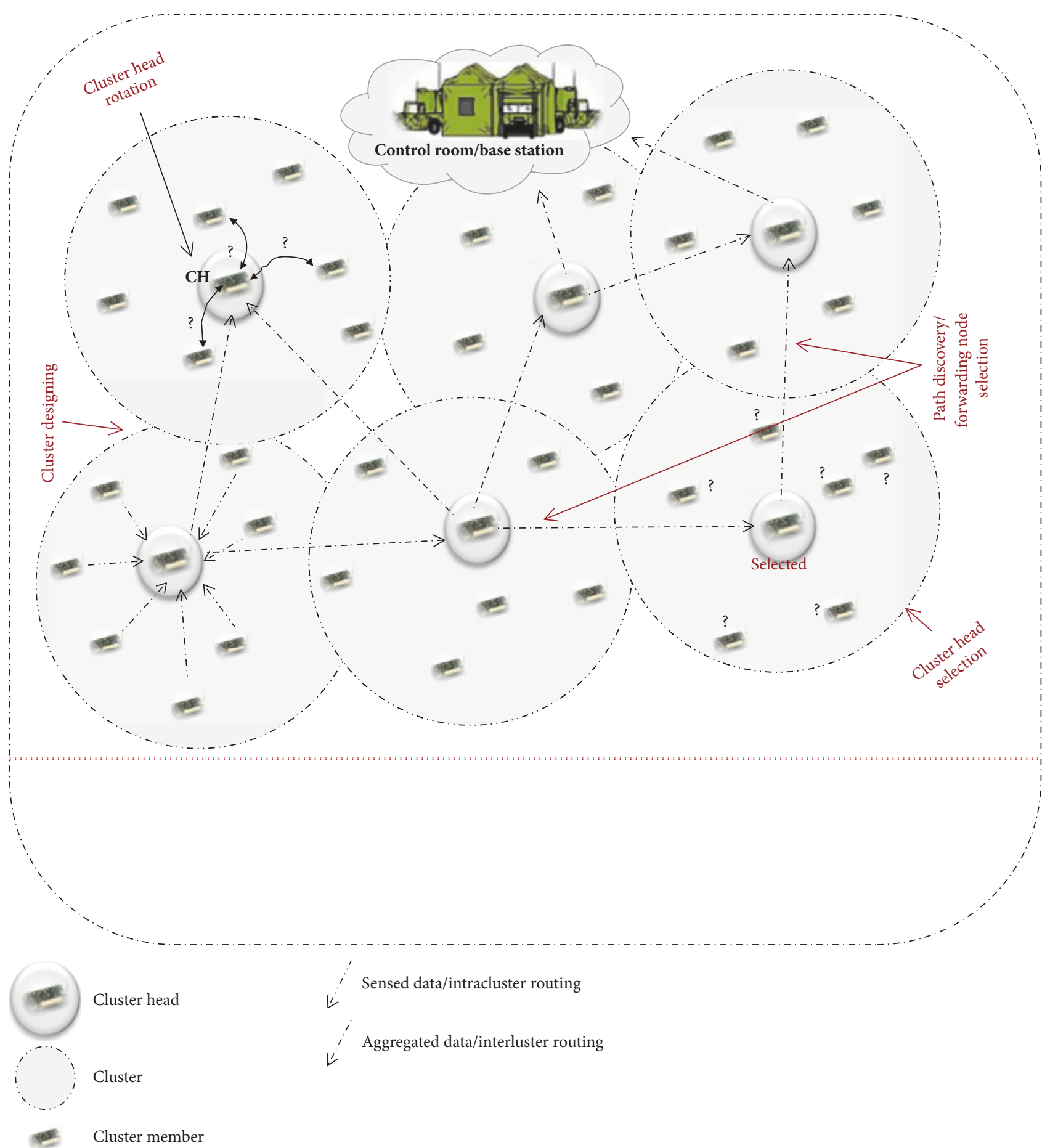

FIGURE 12: Complete scenario of clustered network architecture.

process of cluster designing. There are two methods for the selection of $\mathrm{CH}$ and its members. One is the centralized decision made at the base station that is called centralized cluster designing [86]. The other is distributed decision that is made after exchanging information among neighboring nodes till $\mathrm{CH}$ and its members are selected which is called distributed cluster designing [87]. Both these techniques radically create a mess in energy consumption because of excessive broadcasting especially in large network. It is also due to consistently exchanging messages till the final decision. Thus a hybrid approach exploits the benefits of centralized and distributed cluster designing methodologies and rules out the drawbacks of these techniques. Figure 12 shows the important elements and processes of a clustered network architecture in wireless sensor network. 
TABLE 3: Simulation parameter description.

\begin{tabular}{lc}
\hline Parameter & Description \\
\hline Routing protocols & EADUC, MCDA, E-MCDA (proposed) \\
Simulation area & $500 \mathrm{~m} \times 500 \mathrm{~m}$ and $1000 \mathrm{~m} \times 1000 \mathrm{~m}$ \\
Simulator & NS 2.31 \\
Data rate & 4 packets/sec \\
TCP/IP layer & Network layer \\
Node-to-node distance & Random \\
Node type & Homogenous \\
Number of nodes & 500 \\
Propagation model & Two-ray ground \\
Initial energy of node & 3 joules \\
\hline
\end{tabular}

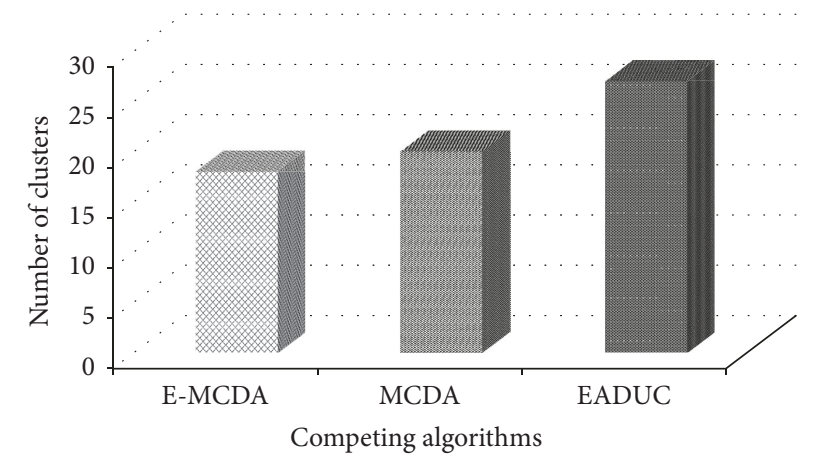

FIGURE 13: Number of designed clusters during cluster formation.

\section{Discussion and Analysis}

We can conclude from the aforesaid discussion that, in the communication process, the functionalities of network layer are of core importance. In this process, the network performance can be improved with energy aware routing. This comes up with increased network lifetime. Flat network architecture and clustered network architecture are two main types of network architecture. These types are based on dissemination of sensed data from source to destination. A sensor network is comprised of hundreds to thousands of nodes that communicate with each other and with base station as well. During exchange of data and information, much energy is consumed with additive issue of intolerable faults and unbalanced load. Flat architecture does not support any conservation of energy by its setup due to undistinguished role among the nodes. In case of clustered architecture, one node is designated as cluster head, and other as member nodes to form a cluster. $\mathrm{CH}$ acquires the sensed data from the cluster member nodes. Next is to aggregate this collected data and forward it to other $\mathrm{CH}$ directly or through multihop or directly to the BS. The routing within the cluster is called intracluster routing, while between the clusters it is named as intercluster. This data communication style leads to significant energy saving. The clustered network is considered to be the most energy efficient architecture due to shortest possible end-to-end delay, ease of route discovery, data aggregation, and fault tolerance nature.
We proposed an energy efficient routing scheme for the multilayer design of wireless sensor networks. It has three major phases, namely, cluster designing, cluster head rotation, and energy aware routing. The first phase has three key constituents: (a) time slot allocation, (b) cluster head selection, and (c) near equal size clusters. Two major outputs in the form of "Multilayer Cluster Designing Algorithm" (MCDA) [48] and "Extended-Multilayer Cluster Designing Algorithm" (E-MCDA) [88] give a detailed working of this phase. MCDA is the initial version while E-MCDA is the extended version as its name implies. Table 3 shows the simulation parameters taken during our experimentations [88].

Some of the results as an example are given in Figures $13,14,15$, and 16 . They show the comparative performance of proposed schemes, that is, E-MCDA and MCDA with each other and with another scheme, an Energy Aware Distributed Unequal Clustering Protocol for Wireless Sensor Networks (EADUC) [89]. Detailed results and comprehensive discussion thereon are available in the articles at $[48,88]$.

In continuation to it, for the second and third phases, that is, cluster head rotation and energy aware routing respective, a scheme called Energy Efficient Strategy for Throughput Improvement in Wireless Sensor Networks (EAR4MCDA) was proposed [90]. This all comes up with the output of network lifetime improvement. Figure 17 shows the phases of three constituents of the proposed scheme. 


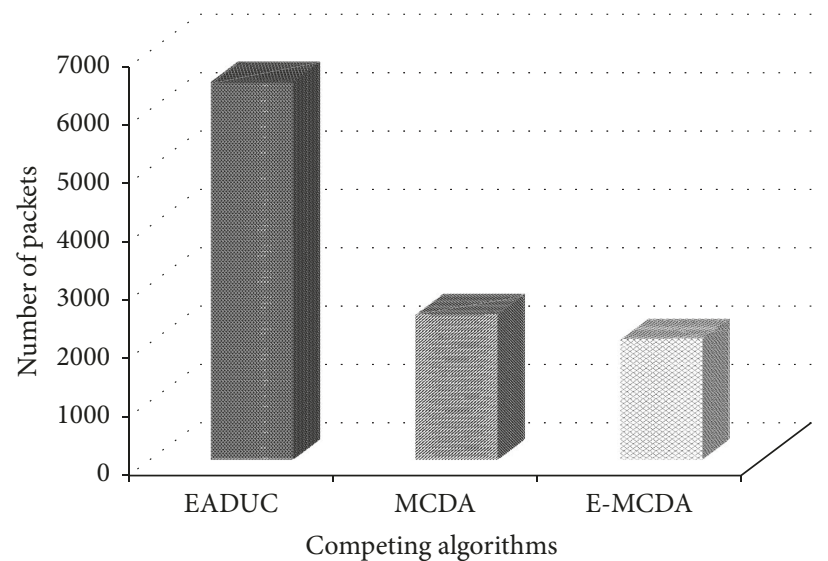

FIGURE 14: Total number of transmissions during cluster formation process.

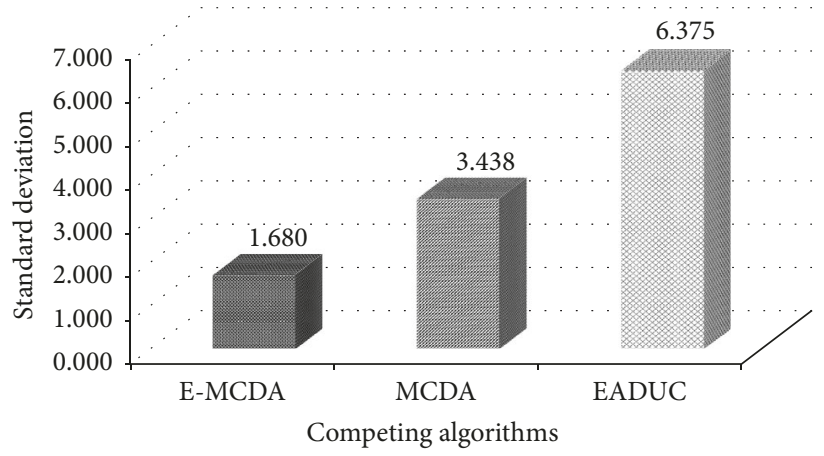

FIGURE 15: Standard deviation of number of cluster members.

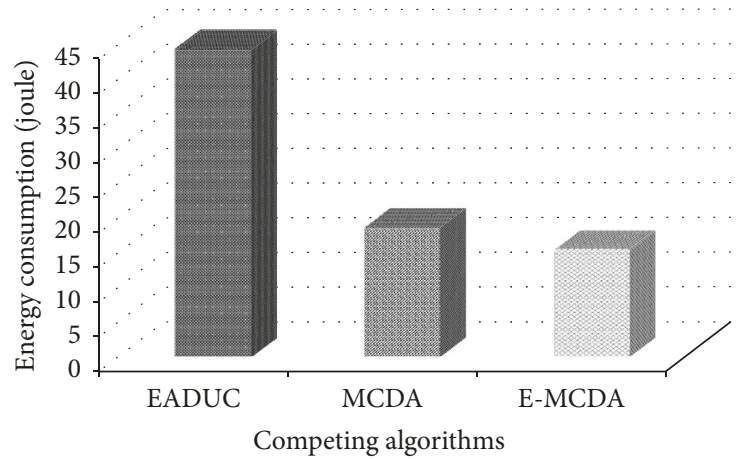

FIGURE 16: Total energy consumption during cluster formation process.

Figures $18,19,20,21,22$, and 23 are giving the comparative performance of proposed scheme, EAR4MCDA with Threshold Based Load Balancing Protocol for Energy Efficient Routing in WSN (TLPER) [91] and EADUC. Detailed results and a comprehensive discussion thereon are given in the article at [90].

As a concluded depiction of the results from the effect of the proposed scheme on overall network lifetime improvement, it is shown in Figure 24.

\section{Conclusion}

Wireless sensor network technology has woven itself into diverse fabric of applications due to its capabilities for involvement in multidisciplinary research and its nourishing from a well-renowned group of researchers and organizations. Digging its multifacet capabilities for efficient performance has also unfolded variety of issues as it is a byproduct, and hence it has a long list of hot issues to attract 


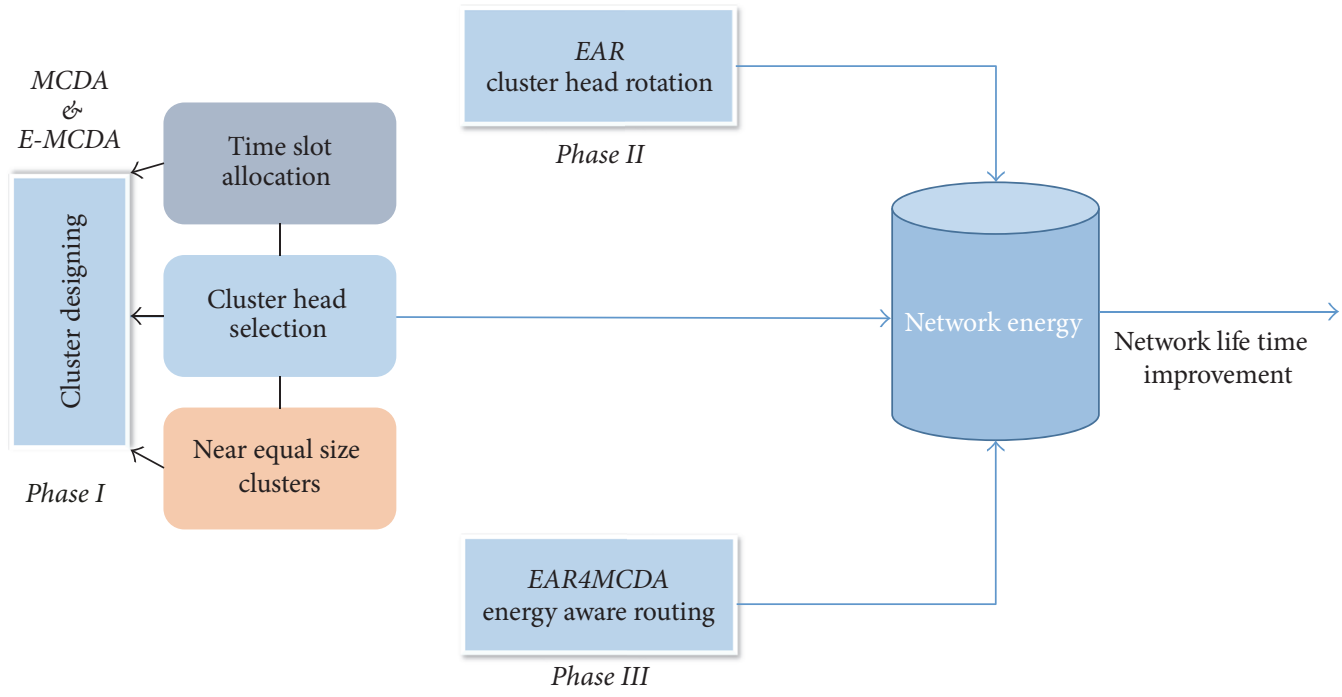

Figure 17: Phases of three constituents of proposed scheme.

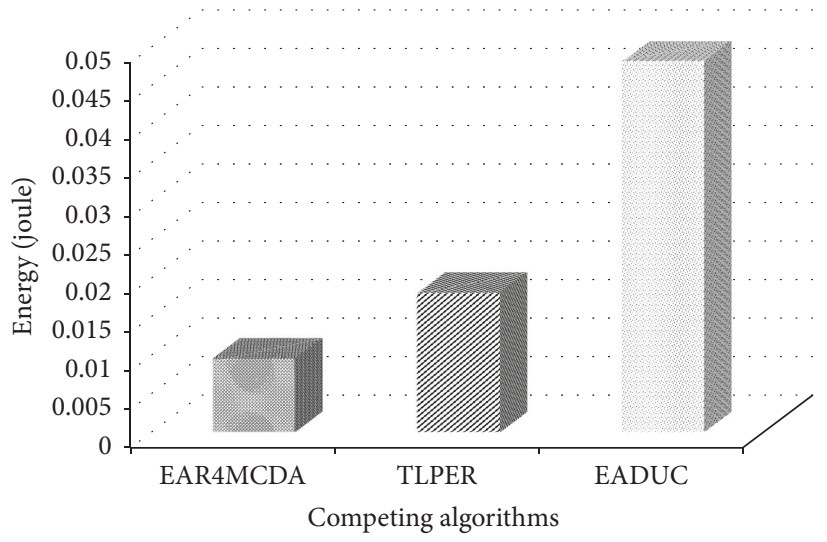

FIGURE 18: Energy consumption in forwarding node selection.

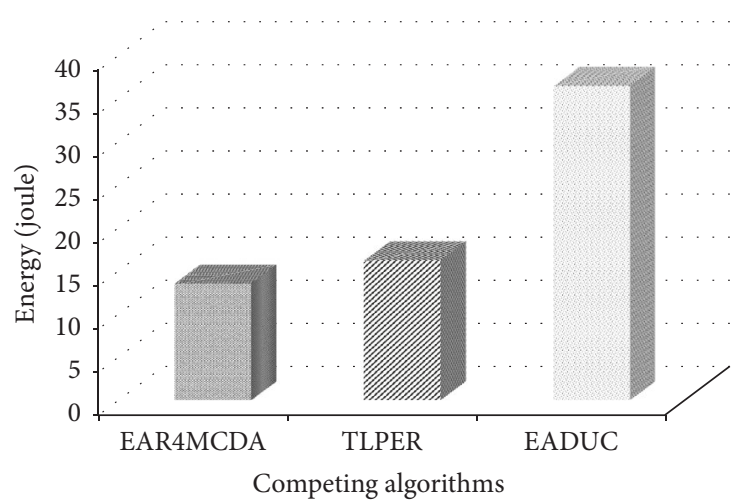

FIGURE 19: Energy consumption during cluster head rotation.

researchers' mind to fix those as is elucidated in Table 2. Its small size is also an appealing reason for its emergence, but it comes with memory, computation, and, the most important, energy constraints. Sensing, transmitting, and receiving are the most energy squeezing acts in the processes of sensor nodes among which transmission is the most energy draining act. These constitute a routing phenomenon to communicate or relaying the sensed data destined to a certain destination. Since the communication is wireless and mostly the deployment of nodes is random, for a well-structured 


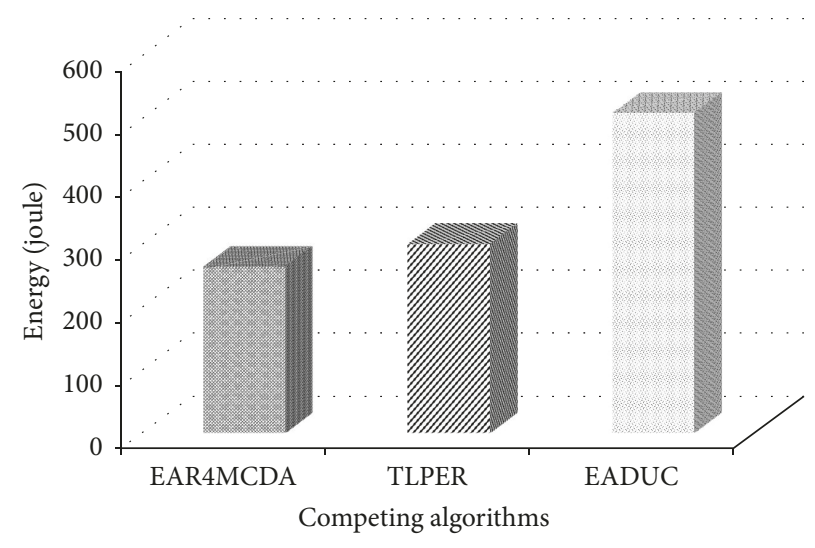

FIGURE 20: Energy consumption against achieved throughput.

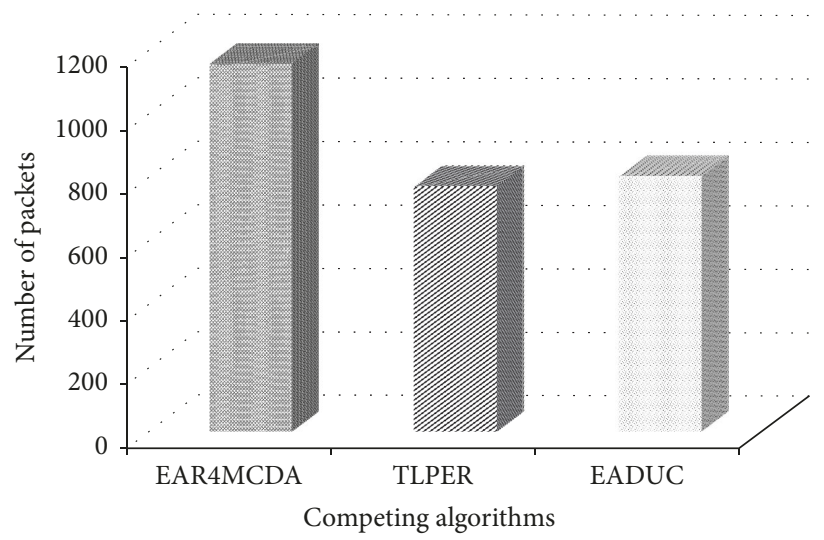

FIgURE 21: Network throughput.

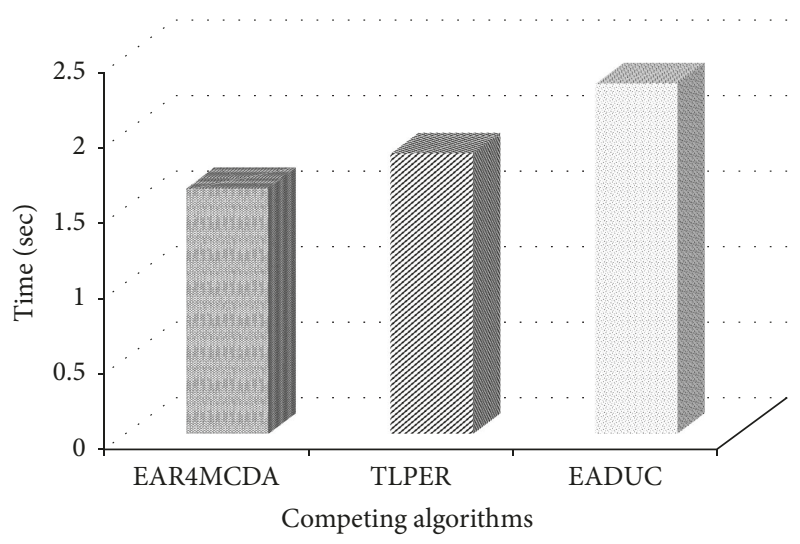

FIGURE 22: End-to-end packet delivery time.

and energy efficient communication, various techniques and algorithms are devised and varied styles of their tuning for optimization are presented in the literature. A well-planned survey of this technique and algorithms with the name of factors affecting the energy aware routing is presented along with their "state-of-the-art" improvement in the literature. If one can have a kick start from this article in routing in WSN, then others can propose comprehensive solution one step ahead of current research on this topic making this article as a good base. These highlighted energy aware factors could be used and exploited in various applications related to the Internet of Things like [92, 93].

\section{Conflicts of Interest}

All the authors confirm that there are no conflicts of interest regarding the publication of this research article. 


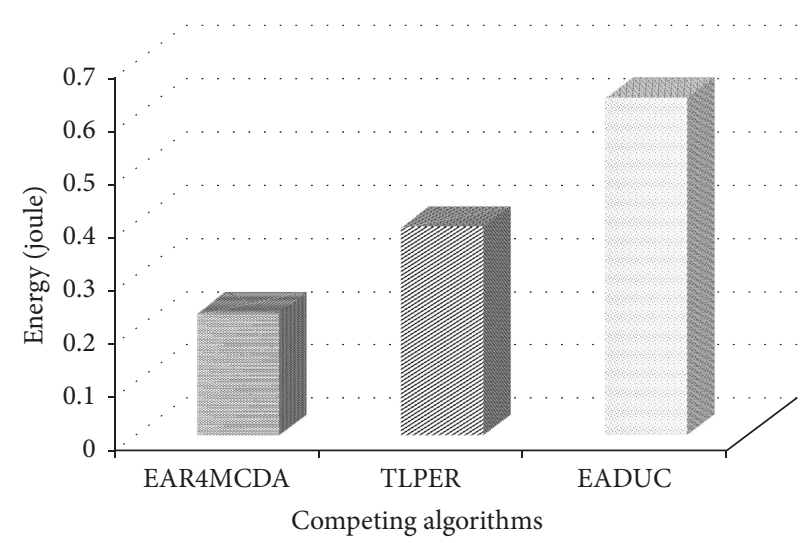

FIGURE 23: Energy consumption for single packet delivery.

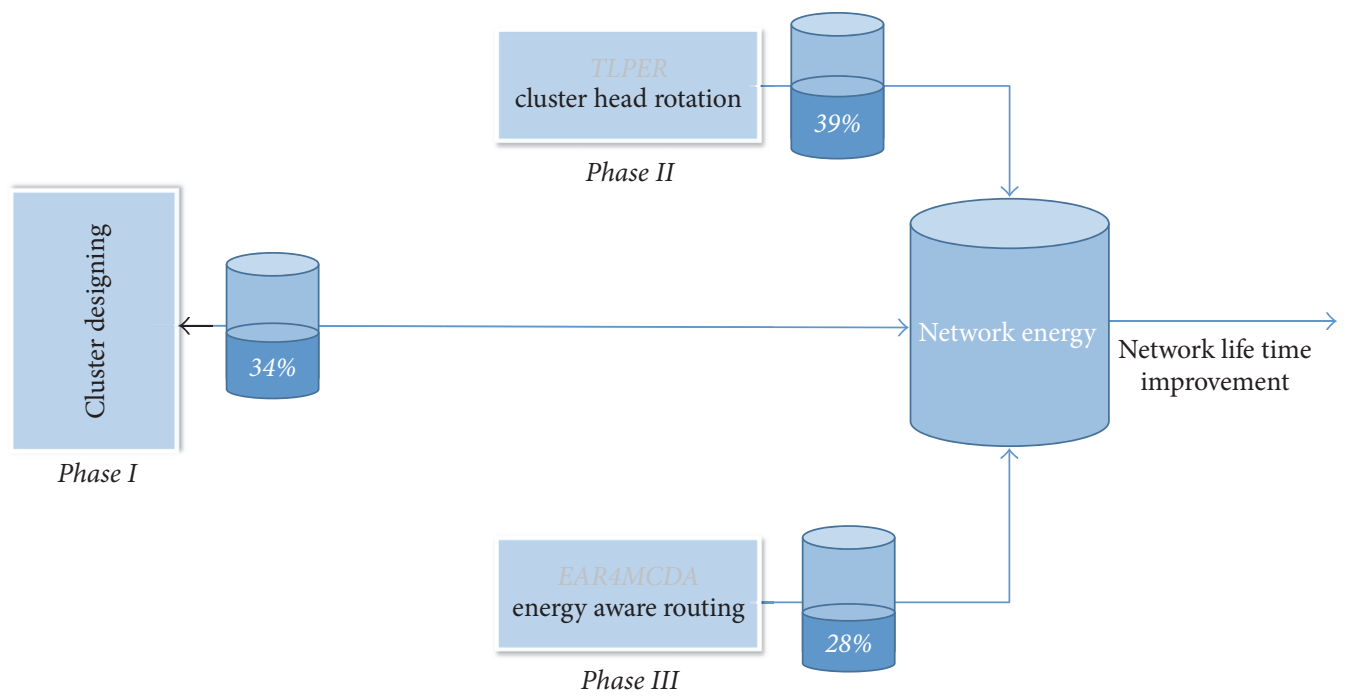

FIGURE 24: Effect of proposed schemes on overall network life time improvement.

\section{Acknowledgments}

(i) This study was supported by the BK21 Plus project (SW Human Resource Development Program for Supporting Smart Life) funded by the Ministry of Education, School of Computer Science and Engineering, Kyungpook National University, Korea (21A20131600005). (ii) This research was supported by Basic Science Research Program through the National Research Foundation of Korea (NRF) funded by the Ministry of Education (2016R1D1A1B03933566). (iii) This work was supported by the Institute for Information \& Communications Technology Promotion (IITP) grant funded by the Korean Government (MSIP) (no. 2017-0-00770).

\section{References}

[1] "MicaZ Data Sheet," Cross bow Technology, Inc., February 2007, http://www.openautomation.net/uploadsproductos/micaz_datasheet.pdf.

[2] S. Jabbar, M. Z. Aziz, A. A. Minhas, and D. Hussain, "PTAL: power tuning anchors localization algorithm for wireless adhoc micro sensors network," in Proceedings of the 7th IEEE
International Conferences on Embedded Software and Systems, Bradford, UK, 2010.

[3] X. Zhu, X. Wu, and G. Chen, "Relative localization for wireless sensor networks with linear topology," Computer Communications, vol. 36, no. 15-16, pp. 1581-1591, 2013.

[4] A. Couteau, F. Evennou, and D. Cibaud, "Anchor-free absolute localization and tracking system for wireless sensor networks," in Proceedings of the IEEE 73rd Vehicular Technology Conference (VTC-Spring '11), Yokohama, Japan, May 2011.

[5] S. Jabbar, A. A. Minhas, R. A. Akhtar, and M. Z. Aziz, "REAR: real-time energy-aware routing for wireless sensor network," in Proceedings of the IEEE International Conference on Pervasive Intelligence and Computing, Chengdu, China, 2009.

[6] M. Ilyas and I. Mahgoub, Handbook of Sensor Networks: Compact Wireless and Wired Sensing Systems, CRC Press, 1st edition, 2004.

[7] S. H. Shah, W. Ali, I. Ahmad, S. Jabbar, and A. A. Minhas, "Performance analysis of queuing mechanism in voice applications," in Proceedings of the World Congress on Engineering, London, UK, 2013.

[8] K. Pister, “Smart Dust,” 2010, http://robotics.eecs.berkeley.edu/ pister/SmartDust/. 
[9] I. Lee, J. Y.-T. Leung, and S. H. Son, Handbook of Real-Time and Embeded Systems, Computer and Information Science, vol. 1, Chapman and Hall/CRC, Boca Raton, Fla, USA, 1st edition, 2008.

[10] "WSN in the real world," ZigBee Alliance, 2011, http://www.automatedbuildings.com/releases/sep11/110929102404zigbee.html.

[11] M. Marin-Perianu, S. Chatterjea, R. Marin-Perianu et al., "Wave monitoring with wireless sensor networks," in Proceedings of the International Conference on Intelligent Sensors, Sensor Networks and Information Processing (ISSNIP '08), Sydney, Australia, December 2008.

[12] A. Alkandari, M. Alnasheet, Y. Alabduljader, and S. M. Moein, "Wireless Sensor Network (WSN) for water monitoring system: case study of Kuwait beaches," International Journal of Digital Information and Wireless Communications, vol. 1, no. 4, pp. 709717, 2011.

[13] M. R. Martonosi, “The Princeton ZebraNet Project: mobile sensor networks for wildlife tracking," 2005, http://www.cis .upenn.edu/departmental/events/abstracts-2004/marg.htm.

[14] K. H. Kwong, T. T. Wu, H. G. Goh et al., "Implementation of herd management systems with wireless sensor networks," IET Wireless Sensor Systems, vol. 1, no. 2, pp. 55-65, 2011.

[15] E. H. Callaway, “NeuRFon,” 1999, http://www.encyclo.co.uk/ define/NeuRFon.

[16] T. Naumowicz, R. Freeman, H. Kirk et al., "Wireless sensor network for habitat monitoring on Skomer Island," in Proceedings of the 5th IEEE International Workshop on Practical Issues in Building Sensor Network Applications, Denver, Colo, USA, 2010.

[17] A. Alkandari, "Traffic surveillance by wireless sensor networks," PATH Research Report, Institute of Transportation Studies, University of California, Berkeley, Calif, USA, 2007.

[18] O. Bondarenko, S. Kininmonth, and M. Kingsford, "Underwater sensor networks, oceanography and plankton assemblages," in Proceedings of the 3rd International Conference on Intelligent Sensors, Sensor Networks and Information, 2007.

[19] A.-J. Garcia-Sanchez, F. Garcia-Sanchez, F. Losilla et al., "Wireless sensor network deployment for monitoring wildlife passages," Sensors, vol. 10, no. 8, pp. 7236-7262, 2010.

[20] R. Kacimi, R. Dhaou, and A.-L. Beylot, "Using energy-efficient wireless sensor network for cold chain monitoring," in Proceedings of the 6th IEEE Consumer Communications and Networking Conference (CCNC '09), January 2009.

[21] A. T. Kunnath and M. V. Ramesh, "Integrating Geophone Network to real-time wireless sensor network system for landslide detection," in Proceedings of the 1st International Conference on Sensor Device Technologies and Applications, July 2010.

[22] A. J. Laflor-Hernández, J. I. Nieto-Hipólito, M. VazquezBriseño, H. C.-D. Avila, J. D. S. López, and E. J. García, "MMIG: a middleware for communicating a WSN to a Web site for monitoring vital signs," in Proceedings of the Pan American Health Care Exchanges Conference, April 2011.

[23] G. Padmavathi, D. Shanmugapriya, and M. Kalaivani, "A study on vehicle detection and tracking using wireless sensor networks," Wireless Sensor Network, vol. 2, no. 2, pp. 173-185, 2010.

[24] A. D. Wood, J. A. Stankovic, G. Virone et al., "Context-aware wireless sensor networks for assisted living and residential monitoring," IEEE Network, vol. 22, no. 4, pp. 26-33, 2008.

[25] C. Wartmann and S. Kohler, "Wireless sensor networks in sick buildings," 2014, http://www.embedded.arch.ethz.ch/Projects/ SickBuilding.
[26] T. Schmid, H. Dubois-Ferrière, and M. Vetterli, "SensorScope: experiences with a wireless building monitoring sensor network," in Proceedings of the 1st Workshop on Real-World Wireless Sensor Networks, Stockholm, Sweden, June 2005.

[27] F. Ingelrest, G. Barrenetxea, G. Schaefer, M. Vetterli, O. Couach, and M. Parlange, "Sensorscope: application-specific sensor network for environmental monitoring," ACM Transactions on Sensor Networks, vol. 6, no. 2, article 17, 2010.

[28] G. Barrenetxea, F. Ingelrest, G. Schaefer, M. Vetterli, O. Couach, and M. Parlange, "SensorScope: out-of-the-box environmental monitoring," in Proceedings of the IEEE International Conference on Information Processing in Sensor Networks (IPSN '08), pp. 332-343, St. Louis, Mo, USA, April 2008.

[29] P. M. Vetterli, "Sensorscope: sensor networks for environmental monitoring," 2013, http://lcav.epfl.ch/sensorscope-en.

[30] D. Rus and E. Basha, Wireless Sensor Network Provides Early Flood Detection for Underserved Countries, Microsoft Reseach, Washington, DC, USA, 2006.

[31] S. H. Wright, "Students tackle flooding in Honduras," 2005, http://newsoffice.mit.edu/2005/honduras.

[32] C. Tschudin, S. Gruber, D. V. Muhll, A. Hasler, and I. Talzi, "PermaSense Project home page," 2006, http://cn.cs.unibas.ch/ projects/permasense/.

[33] I. Talzi, A. Hasler, S. Gruber, and C. Tschudin, "PermaSense: investigating permafrost with a WSN in the Swiss Alps," in Proceedings of the 4th Workshop on Embedded Networked Sensors (EmNets '07), pp. 8-12, ACM, Cork, Ireland, June 2007.

[34] S. Khan, A. Abbas, T. Rana, and J. Carroll, Dairy Water Use in Australian Dairy Farms: Past Trends and Future Prospects, CSIRO: Water for a Healthy Country National Research Flagship, Canberra, Australia, 2010.

[35] M. Ito, Y. Katagiri, M. Ishikawa, and H. Tokuda, "Airy notes: an experiment of microclimate monitoring in shinjuku gyoen garden," in Proceedings of the 4th International Conference on Networked Sensing Systems, pp. 260-266, Braunschweig, Germany, June 2007.

[36] M. Ito, "Airy Notes," 2006, http://www.niya.net/airynotes.html.

[37] G. Tolle, J. Polastre, R. Szewczyk et al., "A macroscope in the redwoods," in Proceedings of the 3rd ACM International Conference on Embedded Networked Sensor Systems (SenSys '05), pp. 51-63, San Diego, Calif, USA, November 2005.

[38] N. G. Yang, "Redwoods go high tech: researchers use wireless sensors to study California's state tree," 2003, http://www.berkeley.edu/news/media/releases/2003/07/28_redwood.shtml.

[39] G. Werner-Allen, K. Lorincz, M. Welsh et al., "Deploying a wireless sensor network on an active volcano," IEEE Internet Computing, vol. 10, no. 2, pp. 18-25, 2006.

[40] H. A. Gurbhej Singh, "Design and architectural issues in wireless sensor networks," International Journal of Advanced Research in Computer Science and Software Engineering, vol. 3, no. 1, 2013.

[41] W. Lingming, Physical Layer and Application Layer Issues of Wireless Sensor Network, The University of Texas at Arlington, Arlington, Tex, USA, 2006.

[42] G. Shankar, T. G. Basavaraju, D. Manjaiah, and K. S. Subir, "Issues in wireless sensor networks," in Proceedings of the World Congress on Engineering, London, UK, 2008.

[43] A. Jain, D. Sharma, M. Goel, and A. K. Verma, "Protocols for network and data link layer in WSNs: a review and open issues," in Advances in Networks and Communications, Communications in Computer and Information Science, vol. 132, pp. 546-555, Springer, 2011. 
[44] I. S. Demirkol, Medium Access Control Layer Performance Issues in Wireless Sensor Network, Boğaziçi University, 2008.

[45] A. A. Alhameed and S. G. Baicher, "MAC layer overview for wireless sensor networks," in Proceedings of the International Conference on Computer Networks and Communication Systems, 2012.

[46] P. R. Gundalwar and V. N. Chavan, "Analysis of Wireless Sensor Networks (WSNs) and routing issues," International Journal of Engineering Research \& Technology, vol. 1, no. 8, 2012.

[47] "Routing security issues in wireless sensor networks: attacks and defenses," in Sustainable Wireless Sensor Networks, pp. 279-309, INTECH Publishers, 2010.

[48] S. Jabbar, A. A. Minhas, A. Paul, and S. Rho, "MCDA: multilayer cluster designing algorithm for network lifetime improvement of homogenous wireless sensor networks," The Journal of Supercomputing, vol. 11227, 2014.

[49] A. Rahman, S. A. El, and W. Gueaieb, "Wireless sensor network transport layer: state of the art," in Sensors, S. Mukhopadhyay and R. Huang, Eds., vol. 21, pp. 221-245, Springer, Berlin, Germany, 2008.

[50] S. Zafar, "A survey of transport layer protocols for wireless sensor networks," International Journal of Computer Applications, vol. 33, no. 1, pp. 44-50, 2011.

[51] L. Wang, Physical Layer and Application Layer Issues of Wireless Sensor Network, The University of Texas at Arlington, 2006.

[52] M. A. Tawhid and H. Mahmood, "Recent trends in wireless sensor networks with applications," International Journal of Distributed Sensor Networks, vol. 2014, Article ID 912574, 4 pages, 2014.

[53] K. Maraiya, K. Kant, and N. Gupta, "Application based study on wireless sensor network," International Journal of Computer Applications, vol. 21, no. 8, pp. 9-15, 2011.

[54] I. F. Akyildiz, D. Pompili, and T. Melodia, "Underwater Acoustic Sensor Networks (UW-ASN)," 2004, http://www.ece .gatech.edu/research/labs/bwn/UWASN/.

[55] M. Hammoudeh and R. Newman, "Information extraction from sensor networks using the Watershed transform algorithm," Information Fusion, vol. 22, pp. 39-49, 2015.

[56] M. Hammoudeh, F. Al-Fayez, H. Lloyd et al., "A wireless sensor network border monitoring system: deployment issues and routing protocols," IEEE Sensors Journal, vol. 17, no. 8, pp. 25722582, 2017.

[57] S. Jabbar, Energy efficient multi-layer design of wireless sensor networks [Ph.D. thesis], Bahria University, Islamabad, Pakistan, 2015.

[58] Y.-B. Ko and N. H. Vaidya, "Location-aided routing (LAR) in mobile ad hoc networks," Wireless Networks, vol. 6, no. 4, pp. 307-321, 2000.

[59] J. A. Stankovic, "Wireless sensor networks," Computer Magazine, vol. 41, no. 10, pp. 92-95, 2008.

[60] A. A. Minhas, Power Aware Routing Protocols for Wireless Ad Hoc Sensor Networks, Graz University of Technology, Graz, Austria, 2007.

[61] S. Jabbar, Energy Aware Real-Time Routing in Wireless Sensor Network, Bahria University, Islamabad, Pakistan, 2009.

[62] D. Zhao, K.-W. Chin, and R. Raad, "Minimizing broadcast latency and redundancy in asynchronous wireless sensor networks," Wireless Networks, vol. 20, no. 3, pp. 345-360, 2014.

[63] J. A. Sanchez, P. M. Ruiz, and R. Marin-Perez, "Beacon-less geographic routing made practical: challenges, design guidelines, and protocols," IEEE Communications Magazine, vol. 47, no. 8, pp. 85-91, 2009.

[64] J. Son and T.-Y. Byun, "A routing scheme with limited flooding for wireless sensor networks," International Journal of Future Generation Communication and Networking, vol. 3, no. 3, 2010.

[65] V. Madhusudhana and S. P. Gururaj, "A survey-an efficient flooding scheme for mitigating broadcast storm problem in wireless sensor network," International Journal of Advanced Research in Computer Engineering \& Technology, vol. 4, no. 5, pp. 2417-2421, 2015.

[66] J. Wu and W. Lou, "Forward-node-set-based broadcast in clustered mobile ad hoc networks," Wireless Communications and Mobile Computing, vol. 3, no. 2, pp. 155-173, 2003.

[67] I. A. Umar, Z. M. Hanapi, A. Sali, and Z. A. Zulkarnain, "FuGeF: a resource bound secure forwarding protocol for wireless sensor networks," Sensors, vol. 16, no. 6, article 943, 20 pages, 2016.

[68] A. Vallimayil, K. M. K. Raghunath, V. R. S. Dhulipala, and R. M. Chandrasekaran, "Role of relay node in Wireless Sensor Network: a survey," in Proceedings of the 3rd International Conference on Electronics Computer Technology, pp. 160-167, Kanyakumari, India, April 2011.

[69] K. Lee and H. Lee, "A self-organized and smart-adaptive clustering and routing approach for wireless sensor networks," International Journal of Distributed Sensor Networks, vol. 2012, Article ID 156268, 13 pages, 2012.

[70] R. A. Akhtar, A. A. Minhas, and S. Jabbar, "Energy aware intra-cluster routing for wireless sensor networks," International Journal for Hybrid Information Technology, vol. 13, no. 1, 2010.

[71] W. R. Heinzelman, A. Chandrakasan, and H. Balakrishnan, "Energy-efficient communication protocol for wireless microsensor networks," in Proceedings of the 33rd Annual Hawaii International Conference on System Siences (HICSS '00), Maui, Hawaii, USA, January 2000.

[72] S. Jabbar, A. A. Minhas, and R. A. Akhtar, "SPERT: a stateless protocol for energy-sensitive real-time routing for wireless sensor network," in Proceedings of the International Conference on Information and Communication Technologies, Karachi, Pakistan, August 2009.

[73] A. A. Minhas, S. Jabbar, M. Zubair Aziz, and W. Mahmood, "QERT: query-based energy-aware real-time routing for wireless sensor network," in Proceedings of the IEEE 10th International Conference on Computer and Information Technology (CIT '10), pp. 2861-2866, Bradford, UK, 2010.

[74] M. Qayyum, S. Jabbar, S. Rizwan, and F. Azam, "Stagnant free ant based energy aware heuristic routing in wireless sensor network," in Proceedings of the 13th International Conference on Collaboration Technologies and Systems (CTS '12), pp. 164-169, Denver, Colo, USA, May 2012.

[75] S. M. Khan, M. M. Khan, and N. A. Khan, "Route constancy and energy aware routing protocol for MANET," International Journal of Computer Science and Network Security, vol. 17, no. 5, pp. 56-64, 2017.

[76] M. Aissani, S. Bouznad, A. Hariza, and S.-E. Allia, "An effective approach for handling both open and closed voids in wireless sensor networks," Sensors \& Transducers Journal, vol. 14, no. 2, pp. 196-210, 2012.

[77] M. Aissani, S. Bouznad, S.-E. Allia, and A. Hariza, "Efficient forwarding approach on boundaries of voids in wireless sensor networks," International Journal on Advances in Telecommunications, vol. 5, no. 3, 2012. 
[78] Q. Fang, J. Gao, and L. J. Guibas, "Locating and bypassing holes in sensor networks," Mobile Networks and Applications, vol. 11, no. 2, pp. 187-200, 2006.

[79] T. He, J. A. Stankovic, C. Lu, and T. F. Abdelzaher, "A spatiotemporal communication protocol for wireless sensor networks," IEEE Transactions on Parallel and Distributed Systems, vol. 16, no. 10, pp. 995-1006, 2005.

[80] M. Chen, T. Kwon, S. Mao, Y. Yuan, and V. C. M. Leung, "Reliable and energy-efficient routing protocol in dense wireless sensor networks," International Journal of Sensor Networks, vol. 4, no. 1-2, pp. 104-117, 2008.

[81] B. A. Bakr and L. Lilien, "LEACH-SM: a protocol for extending wireless sensor network lifetime by management of spare nodes," in Proceedings of the International Symposium of on Modeling and Optimization of Mobile, Ad Hoc, and Wireless Networks (WiOpt '11), Princeton, NJ, USA, May 2011.

[82] S. Jabbar and S. H. Shah, Optimizing throughput using energy efficient data aggregation technique for cluster based wireless sensor network [M.S. thesis], Bahria University Islamabad Campus, Islamabad, Pakistan, 2011.

[83] C.-S. Nam, H.-Y. Cho, and D.-R. Shin, "Efficient path setup and recovery in wireless sensor networks by using the routing table," in Proceedings of the 2nd International Conference on Education Technology and Computer (ICETC '10), pp. V4156-V4159, June 2010.

[84] A. Iwata, C.-C. Chiang, G. Pei, M. Gerla, and T.-W. Chen, "Scalable routing strategies for ad hoc wireless networks," IEEE Journal on Selected Areas in Communications, vol. 17, no. 8, pp. 1369-1379, 1999.

[85] D. N. Sandeep and V. Kumar, "Review on clustering, coverage and connectivity in underwater wireless sensor networks: a communication techniques perspective," IEEE Access, vol. 5, pp. 11176-11199, 2017.

[86] M. M. Zanjireh and H. Larijani, "A survey on centralised and distributed clustering routing algorithms for WSNs," in Proceedings of the IEEE 81st Vehicular Technology Conference (VTC Spring '15), Glasgow, UK, May 2015.

[87] A. Cenedese, M. Luvisotto, and G. Michieletto, "Distributed clustering strategies in industrial wireless sensor networks," IEEE Transactions on Industrial Informatics, vol. 13, no. 1, pp. 228-237, 2017.

[88] S. Jabbar, A. A. Minhas, M. Gohar, A. Paul, and S. Rho, "EMCDA: extended-multilayer cluster designing algorithm for network lifetime improvement of homogenous wireless sensor networks," International Journal of Distributed Sensor Networks, vol. 2015, Article ID 902581, 21 pages, 2015.

[89] J. Yu, Y. Qi, G. Wang, Q. Guo, and X. Gu, "An energy-aware distributed unequal clustering protocol for wireless sensor networks," International Journal of Distributed Sensor Networks, vol. 2011, Article ID 202145, 8 pages, 2011.

[90] S. Jabbar, A. A. Minhas, M. Imran, S. Khalid, and K. Saleem, "Energy efficient strategy for throughput improvement in wireless sensor networks," Sensors, vol. 15, no. 2, pp. 2473-2495, 2015.

[91] S. Jabbar, A. E. Butt, Najm-us-Sehr, and A. A. Minhas, "TLPER: threshold based load balancing protocol for energy efficient routing in WSN," in Proceedings of the 13th International Conference on Advanced Communication Technology (ICACT '11), Seoul, South Korea, 2011.

[92] S. Jabbar, M. Khan, B. N. Silva, and K. Han, "A REST-based industrial web of things' framework for smart warehousing," The Journal of Supercomputing, pp. 1-15, 2016.
[93] A. Ahmad, A. Paul, M. Khan et al., "Energy efficient hierarchical resource management for mobile cloud computing," IEEE Transactions on Sustainable Computing, vol. 2, no. 2, pp. 100112, 2017. 


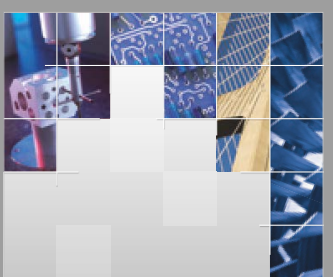

\section{Enfincering}
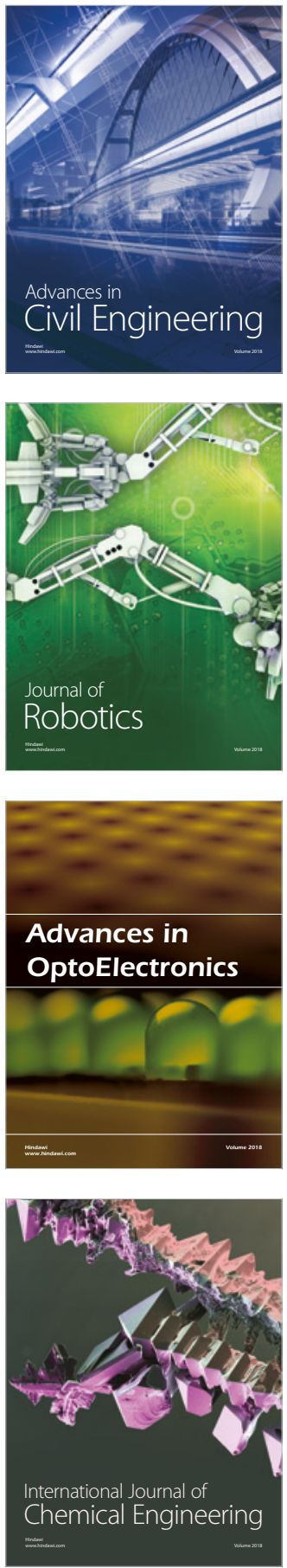

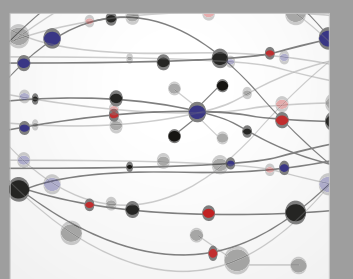

\section{Rotating \\ Machinery}

The Scientific World Journal

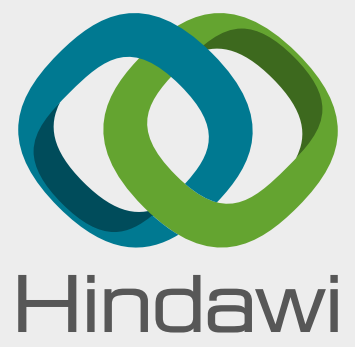

Submit your manuscripts at

www.hindawi.com
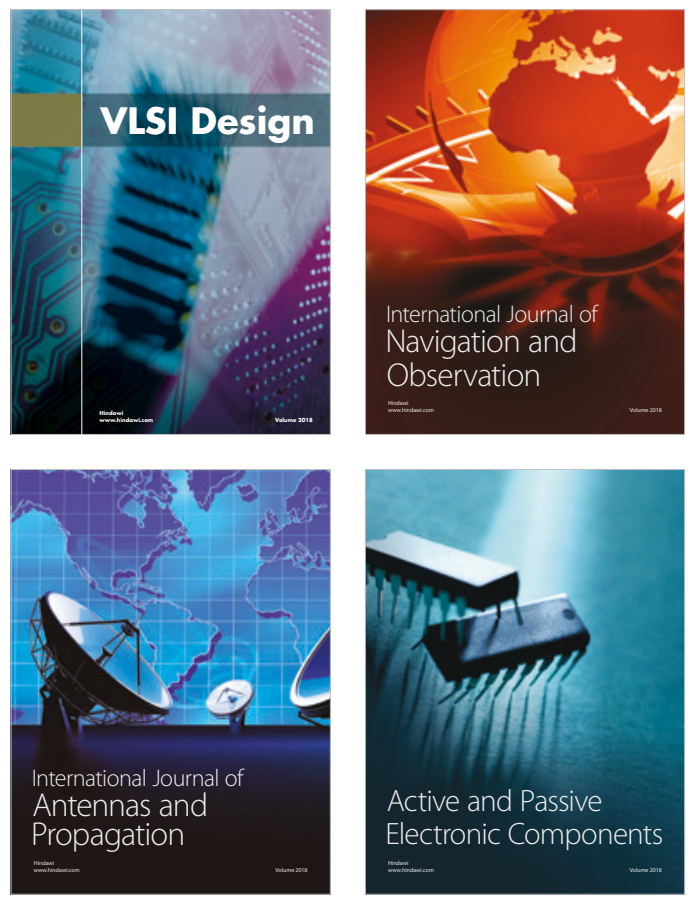
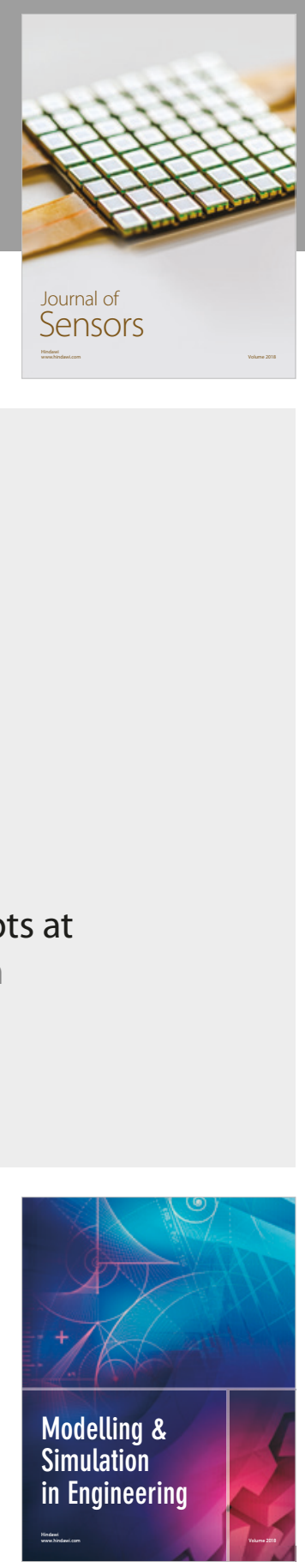

\section{Advances \\ Multimedia}
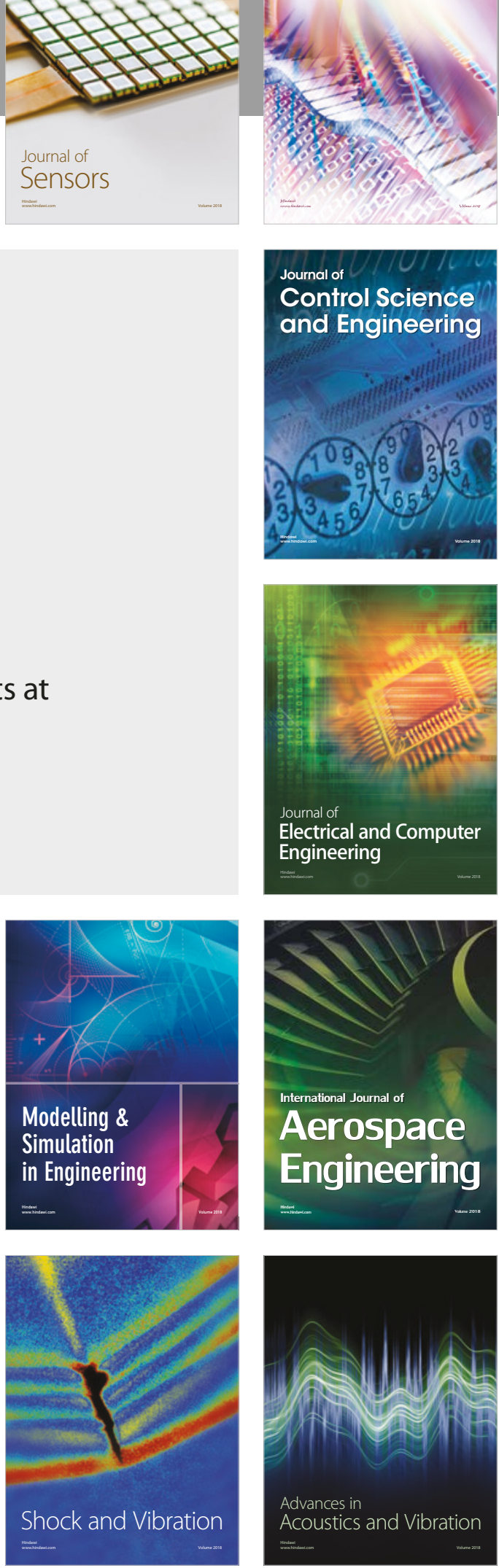